\title{
Synthesis, Antileishmanial Activity and Spin Labeling EPR Studies of Novel $\beta$-Carboline-Oxazoline and $\beta$-Carboline-Dihydrooxazine Derivatives
}

\author{
Paula Baréa, ${ }^{\oplus a}$ Jéssica C. de Paula,${ }^{b}$ Laís Alonso, ${ }^{b, c}$ Aline R. de Oliveira, ${ }^{a}$ Willian F. da Costa,${ }^{a}$ \\ Antonio Alonso, ${ }^{d}$ Celso V. Nakamura ${ }^{b}$ and Maria H. Sarragiotto ${ }^{\circledR} * a$ \\ ${ }^{a}$ Departamento de Química, Universidade Estadual de Maringá (UEM), \\ Av. Colombo, 5790, 87020-900 Maringá-PR, Brazil \\ ${ }^{b}$ Departamento de Análises Clínicas, Universidade Estadual de Maringá(UEM), \\ Av. Colombo, 5790, 87020-900 Maringá-PR, Brazil \\ 'Instituto Federal Goiano, 74663-040 Trindade-GO, Brazil \\ ${ }^{d}$ Instituto de Física, Universidade Federal de Goiás, 74663-040 Goiânia-GO, Brazil
}

\begin{abstract}
A series of novel 1-(substituted-phenyl)-3-(4,5-dihydro-1,3-oxazol-2-yl)-9H- $\beta$-carboline (8a-8i) and 1-(substituted-phenyl)-3-(5,6-dihydro-4H-1,3-oxazin-2-yl)-9H- $\beta$-carboline (9a-9h) derivatives, as well as their respective $N$-(chloroalkyl)-1-(substituted-phenyl)- $9 H$ - $\beta$-carboline-3-carboxamide precursors (6a-6i and $\mathbf{7 a - 7 h})$, were synthesized and evaluated for their in vitro antileishmanial activity against promastigote and intracellular amastigote forms of Leishmania amazonensis. Compounds 8d, 8i, 9e and $\mathbf{9 h}$ exhibited significant activity for both promastigote and amastigote forms, with $\mathrm{IC}_{50}(50 \%$ inhibitory concentration) values ranging from 2.9 to $23.0 \mu \mathrm{M}$. In addition, spin label electron paramagnetic resonance (EPR) spectroscopy studies were carried out for the most active compounds against $L$. amazonensis promastigotes. The studies indicated that the tested compounds cause strong stiffness in the parasite plasma membrane and are capable of inducing internal metalloproteins oxidation of the parasite, resulting in their cross-linking to skeletal proteins. Compounds $8 \mathbf{d}$ and $\mathbf{8 i}$ produced the largest effect, showing that the presence of oxazoline group at $\mathrm{C}-3$ of $\beta$-carboline nucleus is important for antileishmanial activity.
\end{abstract}

Keywords: $\beta$-carboline, 4,5-dihydro-1,3-oxazole, 5,6-dihydro-4H-1,3-oxazine, Leishmania amazonensis, electron paramagnetic resonance

\section{Introduction}

Leishmaniases are a group of diseases caused by protozoan parasites from more than 20 Leishmania species that cause a variety of clinical manifestations in humans, among them, there are three main forms: visceral (VL), cutaneous (CL) and mucocutaneous. ${ }^{1}$ The Leishmania amazonensis species, for example, is responsible for the anergic diffuse cutaneous form and the cutaneous forms with disseminated lesions. ${ }^{2}$ According to World Health Organization (WHO), ${ }^{1} 97$ countries and territories are endemic for leishmaniasis and it is estimated that between 600,000 to 1 million cases of CL occur worldwide annually. The current treatment for the leishmaniasis is mainly performed with

*e-mail: mhsarragiotto@uem.br pentavalent antimonials, amphotericin $\mathrm{B}$, miltefosine and paromomycin. However, there is an increased incidence of treatment failure due to the toxicity and resistance exhibited by these drugs. ${ }^{3}$ Besides this, no vaccines against Leishmania infections are available. ${ }^{4}$ Therefore, it is of great importance to develop more active and less toxic compounds than the drugs used currently.

In the recent years, several studies have reported the antileishmanial activity of heterocyclic compounds, ${ }^{3}$ including $\beta$-carbolines alkaloids. ${ }^{5-20}$ Ashok et al. ${ }^{13}$ for example, described the antileishmanial activity against Leishmania infantum and Leishmania donovani of a series of (1-phenyl-9H-pyrido [3,4-b]indol-3-yl) (4-phenylpiperazin1 -yl)methanone derivatives. Among the derivatives assayed, compound I (Figure 1) displayed potent inhibition for both Leishmania species, with $50 \%$ effective concentration $\left(\mathrm{EC}_{50}\right)$ values ranging from 1.9 to $6.9 \mu \mathrm{M}$. 


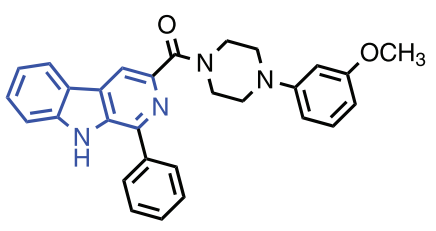

I

potent inhibition for $L$. infantum and L. donovani<smiles>CC(C)(F)Oc1ccc(OC[C@@H]2Cn3cc([N+](=O)[O-])nc3O2)cc1</smiles>

\section{III}

candidate for the oral treatment of visceral leishmaniasis

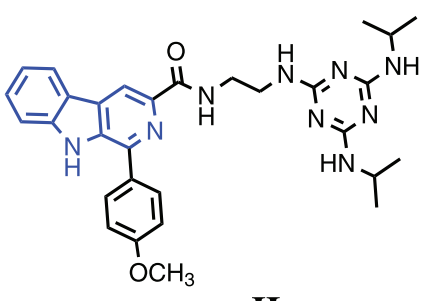

II

potent activity against the promastigote and amastigote forms of $L$. amazonensis

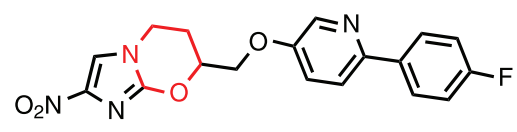

IV

potent activity for $L$. donovani mouse model and $L$. infantum hamster model

Figure 1. Structures of $\beta$-carbolines (I and II), oxazoline (III) and dihydrooxazine (IV) derivatives with antileishmanial activity.

In this context, our research group has already demonstrated the antileishmanial activity of $\beta$-carbolines containing substituents at 1 - and 3-positions of the $\beta$-carboline nucleus. ${ }^{14-20}$ In the works developed by Tonin et al. ${ }^{16}$ and Pedroso et al. ${ }^{17}$ it was demonstrated the activity of $\mathrm{N}$-alkyl-(1-phenylsubstituted$\beta$-carboline)-3-carboxamides against promastigote, axenic amastigote and intracellular amastigote forms of Leishmania amazonensis. The compound with the $\mathrm{N}$-benzyl-carboxamide group at C-3 was active against promastigote and axenic amastigote forms with $\mathrm{IC}_{50}$ (50\% inhibitory concentration) values of 2.6 and $1.0 \mu \mathrm{M}$, respectively, ${ }^{17}$ and killed L. amazonensis promastigotes through different cell death pathways, including apoptosis and autophagy..$^{18}$ Recently, the antileishmanial activity of $\beta$-carboline-1,3,5-triazine hybrids was reported by Baréa et al. ${ }^{19}$ Among the compounds tested, the hybrid II (Figure 1) showed potent activity against the promastigote $\left(\mathrm{IC}_{50}=6.2 \pm 1.4 \mu \mathrm{M}\right.$, selectivity index $\left.(\mathrm{SI})=23.5\right)$ and amastigote $\left(\mathrm{IC}_{50}=1.2 \pm 0.5 \mu \mathrm{M}, \mathrm{SI}=121.4\right)$ forms of $L$. amazonensis and exhibited low toxicity. Studies of action mechanism in promastigotes showed that compound II caused alterations in cell division cycle and an increase of lipid-storage bodies, leading the cells to death through various factors. The accumulation of lipid bodies may be associated with apoptotic cell death. ${ }^{19}$

Additionally, oxazoline and 5,6-dihydro- $4 H$-1,3-oxazine heterocycles play an important role in organic synthesis, being present in the structure of various biologically active compounds. ${ }^{20-27}$ For instance, the nitroimidazo-oxazole III (Figure 1) showed $\mathrm{IC}_{50}$ of $0.03 \mu \mathrm{M}$ against the amastigote form of $L$. donovani DD8 transfected with luciferase, and was identified, from a series of 72 nitroimidazoles evaluated, as a candidate for the oral treatment of visceral leishmaniasis. This compound showed also in vivo activity in both rat and hamster models. ${ }^{25}$ The dihydrooxazine phenylpyridine IV (Figure 1) was effective for $L$. donovani mouse model and $L$. infantum hamster model, displaying optimal efficacy, pharmacokinetic and safety, leading to its selection as a new candidate for treatment of VL. ${ }^{26}$

Considering the promising antileishmanial properties of $\beta$-carboline nucleus, the synthetic and biological importance of oxazoline and 5,6-dihydro- $4 H$-1,3-oxazine rings, and the need to develop antileishmanial agents more effective, in this work we designed new 1-(substituted-phenyl)$\beta$-carboline derivatives bearing oxazoline and 5,6-dihydro$4 H-1,3$-oxazine moieties at C-3 (Figure 2). The novel 1,3-disubstituted- $\beta$-carboline derivatives were evaluated against promastigote and intracellular amastigote forms of $L$. amazonensis and their cytotoxicity were determined. The antileishmanial activity of $N$-(chloroalkyl)- $\beta$-carboline intermediates, precursors of the proposed derivatives, was also evaluated in order to verify the importance of the heterocyclic ring at the 3-position of $\beta$-carboline nucleus.

In addition, electron paramagnetic resonance (EPR) spectroscopy, associated with spin labeling method studies, was carried out for the most active compounds against L. amazonensis promastigotes. This analysis has been shown to be an important tool for analyzing the interaction of drugs or prototypes of drugs with parasite membranes. The literature describes the employment of this technique to evaluate the effects of miltefosine, ${ }^{28,29}$ nerolidol ${ }^{30,31}$ and parthenolide ${ }^{32}$ on L. amazonensis membrane, and of elatol on Trypanosoma cruzi. ${ }^{33}$ 


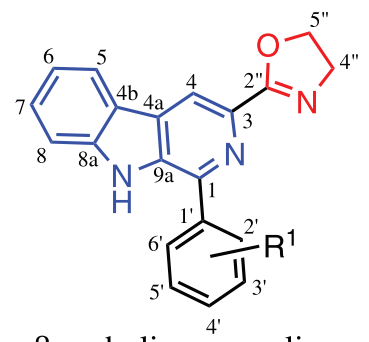

$\beta$-carboline-oxazoline

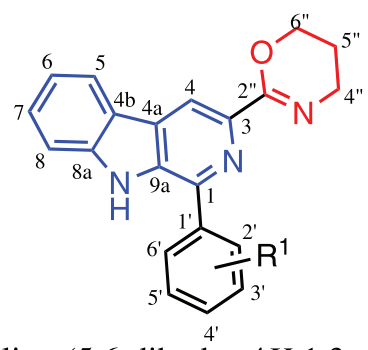

$\beta$-carboline-(5,6-dihydro-4H-1,3-oxazine)

Figure 2. Structures of novel $\beta$-carboline-oxazoline and $\beta$-carboline-(5,6-dihydro- $4 H$-1,3-oxazine) derivatives proposed.

\section{Results and Discussion}

\section{Chemistry}

Novel 1-(substituted-phenyl)- $\beta$-carboline derivatives bearing oxazoline (8a-8i) and 5,6-dihydro- $4 H$-1,3-oxazine $(9 \mathbf{a}-9 \mathbf{h})$ moieties at $\mathrm{C}-3$ were synthesized from the $N$-(chloroalkyl)- $\beta$-carboline-3-carboxamides 6a-6i and $\mathbf{7 a - 7 h}$ as shown in Scheme 1. The $\beta$-carboline nucleus was prepared from commercial L-tryptophan (1) according to the methodology described by our research group. ${ }^{14-19}$ The L-tryptophan methyl ester (2) was subjected to the condensation reaction of Pictet-Spengler with different aldehydes in acid medium, providing the methyl 1-(substituted-phenyl)-1,2,3,4-tetrahydro$9 H$ - $\beta$-carboline-3-carboxylates $\mathbf{3 a - 3 i}$, which were oxidized with sulfur under reflux in xylene to the methyl 1 -(substituted-phenyl)- $9 H$ - $\beta$-carboline-3-carboxylates $\mathbf{4 a}-\mathbf{4} \mathbf{i}$. The basic hydrolysis reaction of $\mathbf{4 a}-\mathbf{4} \mathbf{i}$ provided the 1-(substituted-phenyl)-9H- $\beta$-carboline-3-carboxylic acids $\mathbf{5 a - 5 i}$. The intermediates $\mathbf{5 a - 5 i}$ were subjected to the nucleophilic substitution reaction with 2-chloroethylamine or 3-chloropropylamine, using 4-(4,6-dimethoxy1,3,5-triazin-2-yl)-4-methylmorpholinium chloride (DMTMM) as carboxylic acid activator, providing the $N$-(chloroalkyl)- $\beta$-carbolines $\mathbf{6 a - 6 i}$ and $\mathbf{7 a - 7 i}$ with yields in the range of 52 to $95 \%$.

The $\beta$-carboline-oxazoline $\mathbf{8 a - 8 i}$ derivatives were obtained in $46-93 \%$ yield (Figure 3 ) from the nucleophilic cyclo- $O$-alkylation of $\mathbf{6 a - 6} \mathbf{6}$ in refluxing dimethylformamide (DMF), using potassium carbonate as base. The intramolecular $O$-cyclization of $\mathbf{7 a - 7 h}$ using DMF under microwave irradiation and $\mathrm{K}_{2} \mathrm{CO}_{3}$, afforded the $\beta$-carboline-dihydrooxazine 9a-9h derivatives with yields in the range of 45 to $86 \%$ (Figure 3). The intermediate 7i was also submitted to cyclization reaction, under the similar conditions employed for $\mathbf{7 a - 7 h}$; however, in this case, the product formed underwent decomposition during workup.<smiles>[R]C(=O)C(N)Cc1c[nH]c2ccccc12</smiles>

a

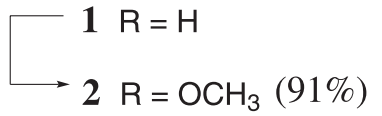

3a-3i<smiles>O=C(N[N+](=O)[O-])c1cc2c3ccccc3[nH]c2c2[nH]c(-c3ccccc3)nc12</smiles>

6a-6i $\mathrm{n}=1$

7a-7i $\mathrm{n}=2$<smiles>[R]c1[R]c(-c2nc(-c3ncco3)cc3c2[nH]c2ccccc23)ccc1</smiles>

8a-8i $n=1$

9a-9h $n=2$
$\left.\mathrm{R}^{1}=\mathrm{a}\right) \mathrm{H}$
b) $2-\mathrm{F}$
c) $4-\mathrm{F}$
d) $2-\mathrm{Cl}$
e) $4-\mathrm{Cl}$ f) $3-\mathrm{NO}_{2}$
g) $4-\mathrm{NO}_{2}$
h) $4-\mathrm{OCH}_{3}$ i) $4-\mathrm{N}(\mathrm{Me})_{2}$

Scheme 1. Synthesis of derivatives 8a-8i and 9a-9h. Reagents and conditions: (a) $\mathrm{MeOH}, \mathrm{H}_{2} \mathrm{SO}_{4}$ (cat), reflux, 48 h; (b) $\mathrm{R}^{1} \mathrm{Ph}-\mathrm{CHO}$, trifluoroacetic acid (TFA), $\mathrm{CH}_{2} \mathrm{Cl}_{2}$, room temperature (rt), 18-26 h; (c) sulfur, xylene, reflux, $48 \mathrm{~h}$; (d) $\mathrm{NaOH}, \mathrm{MeOH} / \mathrm{H}_{2} \mathrm{O}$ (2:1), reflux, 6-18 h; (e) 2-chloroethylamine hydrochloride (for 6a-6i) or 3-chloropropylamine hydrochloride (for 7a-7i), $\mathrm{Et}_{3} \mathrm{~N}$, DMTMM, THF:MeOH (8:2); (f) (i) $\mathrm{K}_{2} \mathrm{CO}_{3}$, DMF, reflux, 12-20 h for 8a-8i; (ii) $\mathrm{K}_{2} \mathrm{CO}_{3}$, DMF, MW (100\% power level), 5-9 min for 9a-9h. 


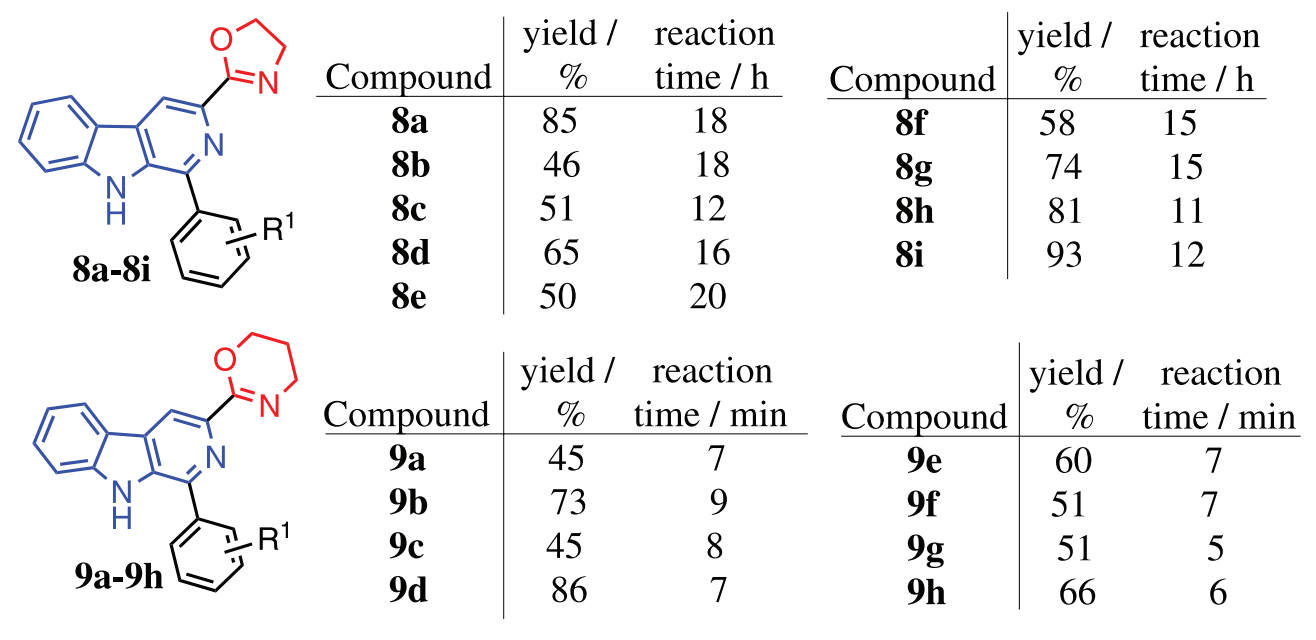
$\left.\mathrm{R}^{1}=\mathrm{a}\right) \mathrm{H}$
b) $2-F$
c) $4-\mathrm{F}$ d) $2-\mathrm{Cl}$
e) $4-\mathrm{Cl}$ f) $3-\mathrm{NO}_{2}$
g) $4-\mathrm{NO}_{2}$
h) $4-\mathrm{OCH}_{3}$ i) $4-\mathrm{N}(\mathrm{Me})_{2}$

Figure 3. Yields and reaction times for preparation of $8 \mathbf{a}-8 \mathbf{i}$ and $9 \mathbf{9}-9 \mathbf{h}$ from cyclo- $O$-alkylation of $6 \mathbf{a}-6 \mathbf{i}$ and $7 \mathbf{a}-7 \mathbf{h}$.

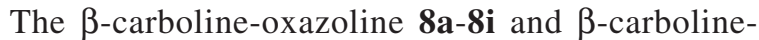
dihydrooxazine $\mathbf{9 a - 9 h}$ derivatives were characterized by their spectral data (high-resolution mass spectrometry (HRMS), ${ }^{1} \mathrm{H}$ and ${ }^{13} \mathrm{C}$ nuclear magnetic resonance (NMR)). The formation of the 8a-8i was supported by the absence of the signal at $\delta_{\mathrm{H}} 8.54-9.01$ referent to the $\mathrm{NH}$ of the carboxamide group and the presence of two triplets at $\left.\delta_{\mathrm{H}} 4.06-4.19\left(\mathrm{CH}_{2}, \mathrm{C}-4\right)\right)$ and 4.50-4.59 $\left(\mathrm{CH}_{2}\right.$, $\mathrm{C}-5$ "), in 'H NMR spectra. The presence of oxazoline ring was also confirmed by the signals at $\delta_{\mathrm{C}}$ 54.4-55.1 $\left(\mathrm{CH}_{2}, \mathrm{C}-4\right.$ ") and 67.4-68.4 $\left(\mathrm{CH}_{2}, \mathrm{C}-5\right.$ "), in ${ }^{13} \mathrm{C}$ NMR spectra. The 5,6-dihydro-4H-1,3-oxazine heterocycle at 3 -positon of $\beta$-carboline nucleus in compounds $\mathbf{9 a - 9 h}$ were confirmed by the signals at $\delta_{\mathrm{H}} 1.93-2.07$ (quintet, $\mathrm{CH}_{2}$ ), 3.55-3.74 (triplet, $\mathrm{CH}_{2}$ ) and 3.83-4.51 (triplet, $\mathrm{CH}_{2}$ ), in ${ }^{1} \mathrm{H}$ NMR spectra, and signals at $\delta_{\mathrm{C}} 21.6-22.0$ $\left(\mathrm{CH}_{2}\right), 42.1-43.0\left(\mathrm{CH}_{2}\right)$ and 64.7-65.7 $\left(\mathrm{CH}_{2}\right)$, in ${ }^{13} \mathrm{C} \mathrm{NMR}$ spectra.

\section{Antileishmanial activity}

The $\beta$-carboline-oxazolines $\mathbf{8 a - 8 i}$ and $\beta$-carbolinedihydrooxazines $9 \mathbf{9}-9 \mathbf{9}$ were evaluated in vitro against the promastigote form of L. amazonensis (Table 1). The compounds that showed $\mathrm{IC}_{50}$ values greater than $100 \mu \mathrm{M}$ were considered inactive. For the most active compounds against promastigotes, the antileishmanial activity for the intracellular amastigote form of $L$. amazonensis was also evaluated. The toxic effects on the host cells were determined by the selectivity index (SI). The SI for each active compound was calculated as the ratio between the cytotoxicity $\left(\mathrm{CC}_{50}\right)$ for macrophage J774-A 1 cell lines and $\mathrm{IC}_{50}$ against the promastigote and intracellular amastigote forms of L. amazonensis.
Analysis of the $\mathrm{IC}_{50}$ values (Table 1 ) for $\beta$-carbolineoxazolines 8a-8i shows that the presence of chlorine and dimethylamino substituents, at 2- and 4-positions of phenyl group linked to $\mathrm{C}-1$, led to the active compounds $\mathbf{8 d}$ and $\mathbf{8 i}$, respectively. Compound $\mathbf{8 d}$ showed also better selectivity indices (SI) for both forms of L. amazonensis than for the host cells (Table 1), being the most promising compound in this series.

Concerning to $9 \mathbf{a}-\mathbf{9 h}$ series, most of $\beta$-carbolinedihydrooxazine derivatives showed moderate activity for L. amazonensis promastigotes, with $\mathrm{IC}_{50}$ values ranging from 21.3 to $58.0 \mu \mathrm{M}$ (Table 1). The derivatives $9 \mathbf{a}$, $\mathbf{9 e}$ and $\mathbf{9 h}$ containing the phenyl, 4-chlorophenyl and 4-methoxyphenyl substituents, respectively, at C-1 of $\beta$-carboline nucleus, were the most active compounds for promastigote form, exhibiting $\mathrm{IC}_{50}$ values in the range of 21.3 to $27.5 \mu \mathrm{M}$, similar to that of reference drug miltefosine. ${ }^{34}$ These compounds were then evaluated against intracellular amastigote form of $L$. amazonensis and showed $\mathrm{IC}_{50}$ values in the range of 2.9 to $75.5 \mu \mathrm{M}$ (Table 1). The derivative $9 \mathbf{e}$ was the most promising, being 26 and 6 times more active than $9 \mathbf{a}$ and $9 \mathbf{h}$, respectively. Besides that, 9e was 29.7 times more toxic for intracellular amastigotes than for macrophage J774-A1 cell lines, being a promising antileishmanial agent.

Comparison of the $\mathrm{IC}_{50}$ data of the novel 1,3-disubstituted$\beta$-carboline derivatives, $\mathbf{8 a - 8 h}$ and $\mathbf{9 a - 9 h}$ showed that except for compound $\mathbf{8 d}$, the replacement of oxazoline by 5,6-dihydro- $4 H-1,3$-oxazine ring led to an enhancement of the antileishmanial activity.

In this work, we also compared the antileishmanial activity of the $\beta$-carboline derivatives $\mathbf{8 a - 8 i}$ and $\mathbf{9 a - 9 h}$ with their intermediates $N$-(chloroalkyl)- $\beta$-carboline 6a-6i and 7a-7h, respectively (Table 2). Among the $N$-(chloroalkyl)- 
Table 1. Antileishmanial activity data for derivatives $\mathbf{8 a - 8 i}$ and $\mathbf{9 a - 9 h}$ against L. amazonensis

\begin{tabular}{|c|c|c|c|c|c|}
\hline Compound & $\mathrm{IC}_{50 \mathrm{pro}} / \mu \mathrm{M}$ & $\mathrm{IC}_{50 \mathrm{ama}} / \mu \mathrm{M}$ & $\mathrm{CC}_{50} / \mu \mathrm{M}$ & $\mathrm{SI}_{\text {pro }}$ & $\mathrm{SI}_{\text {ama }}$ \\
\hline $\mathbf{8 a}$ & $>100$ & n.t & $270.0 \pm 28.3$ & n.d & n.d \\
\hline $8 b$ & $>100$ & n.t & $112.5 \pm 24.7$ & n.d & n.d \\
\hline $8 c$ & $>100$ & n.t & $95.0 \pm 5.0$ & n.d & n.d \\
\hline $8 d$ & $14.7 \pm 6.7$ & $10.5 \pm 3.2$ & $96.5 \pm 10.6$ & 6.6 & 9.2 \\
\hline $8 \mathrm{e}$ & $>100$ & n.t & $43.0 \pm 18.4$ & n.d & n.d \\
\hline $8 f$ & $>100$ & n.t & $170.5 \pm 28.9$ & n.d & n.d \\
\hline $8 g$ & $>100$ & n.t & $88.0 \pm 10.6$ & n.d & n.d \\
\hline $8 \mathrm{~h}$ & $88.5 \pm 10.6$ & n.t & $249.5 \pm 13.5$ & 2.9 & n.d \\
\hline $8 i$ & $23.0 \pm 7.1$ & $12.3 \pm 3.1$ & $30.5 \pm 5.3$ & 1.3 & 2.5 \\
\hline $9 a$ & $27.5 \pm 6.4$ & $75.5 \pm 6.7$ & $67.5 \pm 17.7$ & 2.5 & 0.9 \\
\hline $9 b$ & $53.5 \pm 17.7$ & n.t & $73.2 \pm 16.3$ & 1.4 & n.d \\
\hline $9 \mathrm{c}$ & $57.3 \pm 11.2$ & n.t & $120.0 \pm 35.0$ & 2.1 & n.d \\
\hline 9d & $58.0 \pm 2.8$ & n.t & $62.8 \pm 24.7$ & 1.1 & n.d \\
\hline $9 e$ & $22.7 \pm 2.1$ & $2.9 \pm 0.8$ & $86.0 \pm 12.1$ & 3.8 & 29.7 \\
\hline $9 f$ & $>100$ & n.t & $95.3 \pm 30.7$ & n.d & n.d \\
\hline $9 \mathrm{~g}$ & $>100$ & n.t & $58.0 \pm 13.8$ & n.d & n.d \\
\hline $9 \mathrm{~h}$ & $21.3 \pm 4.2$ & $17.0 \pm 1.4$ & $39.5 \pm 3.55$ & 1.9 & 2.3 \\
\hline Miltefosine $^{a}$ & $18.5 \pm 1.1$ & $2.4 \pm 0.1$ & $40.5 \pm 1.7$ & 2.2 & 16.9 \\
\hline
\end{tabular}

aPositive control. ${ }^{34} \mathrm{IC}_{50 \mathrm{pro}}: 50 \%$ inhibitory concentration against promastigotes; $\mathrm{IC}_{50 \mathrm{ama}}: 50 \%$ inhibitory concentration against intracellular amastigotes; $\mathrm{CC}_{50}: 50 \%$ cytotoxic concentration; $\mathrm{SI}_{\text {pro }}$ : selectivity index for promastigotes; $\mathrm{SI}_{\text {ama }}$ : selectivity index for intracellular amastigotes; n.t: not tested; n.d: not determined.

$\beta$-carbolines evaluated, only $\mathbf{6 d}$ was active, showing significant activity against both forms of $L$. amazonensis $\left(\mathrm{IC}_{50 \mathrm{pro}}=2.2 \pm 1.3 \mu \mathrm{M} ; \mathrm{IC}_{50 \mathrm{ama}}=6.3 \pm 0.8 \mu \mathrm{M}\right)$. These results demonstrate that the presence of oxazoline and dihydrooxazine rings in the 3 -position of the $\beta$-carboline nucleus is important for antileishmanial activity.

\section{Spin label EPR spectroscopy studies}

In order to investigate the interaction of the most active compounds for the promastigote form of L. amazonensis with the parasite membrane, EPR spectroscopy associated with the spin labeling method studies were carried out for 6d, 8d, 8i, 9a, 9e and 9h. Figure 4 shows the EPR spectra of the spin label 5-doxyl-stearic acid (5-DSA) incorporated in Leishmania membranes for samples untreated and treated with the studied compounds. EPR spectra showed that all compounds cause increases in parameter $2 \mathrm{~A}_{/ /}$(outer hyperfine splitting) above the estimated experimental error $(0.5 \mathrm{G})$, indicating decreases in molecular dynamics. In the treatment with $150 \mu \mathrm{M}$ of compounds, some of them showed remarkable changes in the parasite membrane. Compounds $8 \mathbf{d}$ and $\mathbf{8 i}$ containing the oxazoline heterocycle at 3-position of $\beta$-carboline nucleus were the most effective for treatments at a concentration of $150 \mu \mathrm{M}$. However, we note that the compounds $6 \mathbf{d}, 9 \mathrm{a}, 9 \mathrm{e}$ and $9 \mathrm{~h}$ can also cause high membrane stiffness at higher concentrations. In cell membrane the probe 5-DSA behaves as annular or boundary lipids that preferentially surround the hydrophobic surface of membrane proteins. ${ }^{35}$ Because of these interactions with the transmembrane proteins, 5-DSA can monitor the dynamics at the periphery of proteins into the lipid bilayer. Thus, the changes in 5-DSA spectra caused by the compounds may be associated with changes in the membrane protein component.

Spin label EPR spectroscopy indicated that the treatments of $L$. amazonensis promastigotes with the studied compounds cause strong stiffness in the parasite plasma membrane. These strong membrane changes, with changes in parameter $2 \mathrm{~A}_{/ /}$of ca. $5 \mathrm{G}$ observed for two compounds at a relatively low concentration, cannot be explained by the simple presence of the molecules in the membrane, but must involve some oxidation process. Similar alterations in the EPR spectra of 5-DSA into plasma membrane were found in a previous study ${ }^{36}$ for erythrocytes oxidized with hydrogen peroxide in phosphate buffer with azide (catalase inhibitor). EPR spectra of erythrocyte membrane spin-labeled with 5-DSA showed a change in parameter $2 \mathrm{~A}_{/ /}$of $57.5 \mathrm{G}$ for untreated erythrocyte (control) to $60.5 \mathrm{G}$ for erythrocyte treated with $200 \mu \mathrm{M} \mathrm{H} \mathrm{H}_{2} \mathrm{O}_{2}$. It 
Table 2. Antileishmanial activity data for compounds $6 \mathbf{a}-6 \mathbf{i}$ and $7 \mathbf{a}-7 \mathbf{i}$ against $L$. amazonensis

\begin{tabular}{|c|c|c|c|c|c|}
\hline Compound & $\mathrm{IC}_{50 \mathrm{pro}} / \mu \mathrm{M}$ & $\mathrm{IC}_{50 \mathrm{ama}} / \mu \mathrm{M}$ & $\mathrm{CC}_{50} / \mu \mathrm{M}$ & $\mathrm{SI}_{\text {pro }}$ & $\mathrm{SI}_{\text {ama }}$ \\
\hline $6 a$ & $>100$ & n.t & $175.0 \pm 35.4$ & n.d & n.d \\
\hline $6 b$ & $>100$ & n.t & $49.6 \pm 16.3$ & n.d & n.d \\
\hline $6 c$ & $>100$ & n.t & $161.7 \pm 7.6$ & n.d & n.d \\
\hline $6 d$ & $2.2 \pm 1.3$ & $6.3 \pm 0.8$ & $19.0 \pm 6.2$ & 8.7 & 3.0 \\
\hline $6 e$ & $>100$ & n.t & $127.0 \pm 32.5$ & n.d & n.d \\
\hline $6 f$ & $>100$ & n.t & $201.0 \pm 9.9$ & n.d & n.d \\
\hline $6 \mathrm{~g}$ & $>100$ & n.t & $97.0 \pm 4.2$ & n.d & n.d \\
\hline $6 \mathrm{~h}$ & $>100$ & n.t & $68.5 \pm 10.6$ & n.d & n.d \\
\hline $6 i$ & $>100$ & n.t & $30.0 \pm 3.0$ & n.d & n.d \\
\hline $7 a$ & $>100$ & n.t & $630.0 \pm 169.0$ & n.d & n.d \\
\hline $7 b$ & $92.0 \pm 4.05$ & n.t & $83.3 \pm 20.5$ & n.d & n.d \\
\hline $7 c$ & $>100$ & n.t & $70.7 \pm 16.2$ & n.d & n.d \\
\hline $7 d$ & $>100$ & n.t & $<10$ & n.d & n.d \\
\hline $7 e$ & $>100$ & n.t & $365.0 \pm 120.2$ & n.d & n.d \\
\hline $7 f$ & $>100$ & n.t & $273.3 \pm 59.6$ & n.d & n.d \\
\hline $7 \mathrm{~g}$ & $>100$ & n.t & $895.0 \pm 148.0$ & n.d & n.d \\
\hline $7 \mathrm{~h}$ & $>100$ & n.t & $290.0 \pm 88.2$ & n.d & n.d \\
\hline $7 i$ & $>100$ & n.t & $615.0 \pm 49.5$ & n.d & n.d \\
\hline Miltefosine $^{a}$ & $18.5 \pm 1.1$ & $2.4 \pm 0.1$ & $40.5 \pm 1.7$ & 2.2 & 16.9 \\
\hline
\end{tabular}

Positive control; $;{ }^{34} \mathrm{IC}_{50 \mathrm{pro}}: 50 \%$ inhibitory concentration against promastigotes; $\mathrm{IC}_{50 \mathrm{ama}}: 50 \%$ inhibitory concentration against intracellular amastigotes; $\mathrm{CC}_{50}: 50 \%$ cytotoxic concentration; $\mathrm{SI}_{\text {pro }}$ : selectivity index for promastigotes; $\mathrm{SI}_{\text {ama }}$ : selectivity index for intracellular amastigotes; n.t: not tested; n.d: not determined.

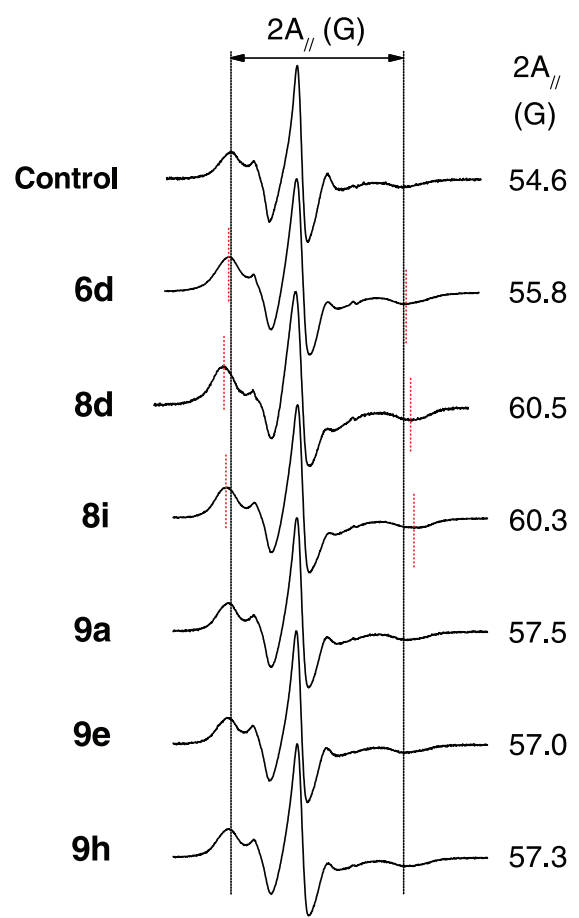

Figure 4. EPR spectra of spin label 5-DSA incorporated in Leishmania amazonensis promastigotes membranes for samples of untreated cells (control) and treated with several compounds $(150 \mu \mathrm{M})$. The values of the EPR parameter $2 \mathrm{~A}_{/ /}$(outer hyperfine splitting), which is given by the separation in magnetic field units between the first peak and the last inverted peak of the spectrum, are indicated. The estimated experimental error for the $2 \mathrm{~A}_{/ /}$parameter is $0.5 \mathrm{G}$. The total scan range of the magnetic field in each EPR spectrum was of $100 \mathrm{G}$ ( $x$ axis) and the intensity ( $y$ axis) is in arbitrary units. has been shown that $\mathrm{H}_{2} \mathrm{O}_{2}$ induces the formation of crosslinking of hemoglobin to skeletal proteins in the membranes of human erythrocytes in an azide phosphate buffer, associated with a progressive alteration of the cell's shape to echinocytic morphology, decreased cell deformability and increased phagocytosis. ${ }^{37}$ Heme proteins were crucial for the occurrence of these cellular alterations, since they may be completely inhibited by previous exposure of red blood cells to carbon monoxide. Lipid peroxidation did not appear to be important because the antioxidant butylated hydroxytoluene decreased the fluorescent derivatives but did not prevent formation of the spectrin-Hb (hemoglobin) complex. ${ }^{37}$ These observations suggest that the compounds tested are capable of inducing oxidation of internal metalloproteins of the parasite, resulting in their crosslinking to skeletal proteins.

\section{Conclusions}

Novel $\beta$-carboline-oxazoline 8a-8i and $\beta$-carbolinedihydrooxazine 9a-9h derivatives have been synthesized in moderate to good yields (45-93\%) from the nucleophilic cyclo- $O$-alkylation of intermediates $N$-(chloroalkyl)$\beta$-carboline-3-carboxamide 6a-6i and 7a-7h, respectively. Compounds 6d, 8d, 8i, 9e and 9 h showed significant activity in $L$. amazonensis promastigotes $\left(\mathrm{IC}_{50}\right.$ values ranging from 2.2 to $23.0 \mu \mathrm{M}$ ), and also in intracellular 
amastigote forms $\left(\mathrm{IC}_{50}\right.$ values ranging from 2.9 to $17.0 \mu \mathrm{M}$ ). Compound $\mathbf{9 e}$ was the most active for intracellular amastigotes $\left(\mathrm{IC}_{50}=2.9 \pm 0.8 \mu \mathrm{M}\right)$ and showed also low toxicity, being 29.7 times more toxic for intracellular amastigotes than for macrophage J774-A1 cell lines.

The antileishmanial activity data showed that the presence of oxazoline and 5,6-dihydro- $4 \mathrm{H}-1,3$-oxazine moieties at $\mathrm{C}-3$ of $\beta$-carboline nucleus led to an increase of compounds number with antileishmanial activity, in comparison to the $N$-(chloroalkyl)- $\beta$-carboline3-carboxamide precursors. Comparison of the $\mathrm{IC}_{50}$ data of the compounds $\mathbf{8 a - 8 h}$ and $\mathbf{9 a - 9 h}$ showed that except for compound $\mathbf{8 d}$, the replacement of oxazoline by 5,6-dihydro-4H-1,3-oxazine ring led to an enhancement of the antileishmanial activity.

Spin label EPR spectroscopy studies indicated that the tested compounds cause strong stiffness in the parasite plasma membrane and are capable of inducing internal metalloproteins oxidation of the parasite, resulting in their cross-linking to skeletal proteins. Compounds $\mathbf{8 d}$ and $\mathbf{8 i}$ produced the largest effect, showing that the presence of oxazoline group at $\mathrm{C}-3$ of $\beta$-carboline nucleus is important for antileishmanial activity. Further studies will be conducted with these compounds aiming a better understanding of their mechanisms of action. Compounds $\mathbf{8 d}$ and $\mathbf{8 i}$ are also strong candidates for in vivo studies in view to the development of new antileishmanial agents.

\section{Experimental}

\section{General methods}

All reagents were purchased from commercial suppliers, except the DMTMM that was synthesized according to the methodology described by Cronin et al. ${ }^{38}$ and Kunishima et al..$^{39}$ The reactions were monitored by thin layer chromatography (TLC) conducted on Whatman TLC plates (silica gel $60 \mathrm{~F}_{254}$ ). NMR spectra were recorded in a Varian spectrometer model Mercury plus BB at 300 (for ${ }^{1} \mathrm{H}$ ) and $75 \mathrm{MHz}$ (for ${ }^{13} \mathrm{C}$ ) and in a Bruker spectrometer model Avance III HD at 500 (for ${ }^{1} \mathrm{H}$ ) and $125 \mathrm{MHz}$ (for $\left.{ }^{13} \mathrm{C}\right)$, with deuterated solvents, chloroform $\left(\mathrm{CDCl}_{3}\right)$, methanol $\left(\mathrm{CD}_{3} \mathrm{OD}\right)$ and dimethyl sulfoxide (DMSO- $\left.d_{6}\right)$, and tetramethylsilane (TMS) as internal standard. Mass spectra (electrospray ionization mass spectrometry (ESI-MS)) were recorded on Thermoelectron Corporation Focus-DSQ II spectrometer. Melting points were determined in Microquímica apparatus model MQAPF-301 and are uncorrected. Spin label 5-DSA was purchased from Sigma-Aldrich (St. Louis, MO, USA).

\section{Chemistry}

\section{Synthesis of $\mathbf{5 a - 5 i}$}

The intermediates 5a-5i were synthesized according to the protocol described previously by our research group. ${ }^{16,17,19}$ To a suspension of 1-(substituted-phenyl)$\beta$-carboline-3-carboxylates $4 \mathbf{a}-4 \mathbf{i}(1 \mathrm{mmol})$ in methanolwater (2:1), it was added $4 \mathrm{mmol}$ of sodium hydroxide $(0.16 \mathrm{~g})$. The mixture was refluxed until complete consumption of $\mathbf{4 a - 4 i}(6-18 \mathrm{~h})$. Then, the reaction mixture was cooled, treated with an $\mathrm{HCl}$ solution (2 M) until pH 5 and left on ice bath for $2 \mathrm{~h}$. The precipitate formed was filtered and washed with distilled water. The $\beta$-carbolinecarboxylic acids $\mathbf{5 a}-\mathbf{5 i}$ were obtained in yields in the range $59-97 \%$.

Synthesis of $\mathrm{N}$-(2-chloroethyl)-1-(substitutedphenyl)-9H- $\beta$-carboline-3-carboxamides (6a-6i) and $N$-(3-chloropropyl)-1-(substituted-phenyl)-9H- $\beta$-carboline3-carboxamides (7a-7i)

To a solution of $\beta$-carboline-carboxylic acids $\mathbf{5 a - 5 i}$ $(1 \mathrm{mmol})$ in tetrahydrofuran/methanol 8:2 $(10 \mathrm{~mL})$, it was added $1.5 \mathrm{mmol}$ of 2-chloroethylamine hydrochloride $(0.17 \mathrm{~g})$ or 3-chloropropylamine hydrochloride $(0.20 \mathrm{~g})$, $1.5 \mathrm{mmol}$ of triethylamine $(0.21 \mathrm{~mL})$ and $1.5 \mathrm{mmol}$ of DMTMM $(0.36 \mathrm{~g})$. The reaction mixture was stirred at room temperature until complete consumption of $\mathbf{5 a - 5 i}$ (14 to $20 \mathrm{~h}$ ). After this reaction time, the solvent was removed in a rotary evaporator and the residue solubilized in $1 \mathrm{~mL}$ of ethanol. Then, distilled water was added to the solution and the precipitate formed was filtered under vacuum and washed with distilled water. The compounds $\mathbf{6 a - 6 i}$ and $7 \mathbf{a}-7 \mathbf{i}$ were obtained in yields in the range of $52-95 \%$.

$N$-(2-Chloroethyl)-1-phenyl-9H- $\beta$-carboline-3-carboxamide (6a)

Yield: 67\%; mp 177-180 ${ }^{\circ} \mathrm{C} ;{ }^{1} \mathrm{H}$ NMR (300 MHz, $\left.\mathrm{CDCl}_{3}\right) \delta_{\mathrm{H}} 3.76(\mathrm{t}, J 5.8 \mathrm{~Hz}, 2 \mathrm{H}, \mathrm{H}-2$ '”), 3.87 (q, J $5.8 \mathrm{~Hz}$, 2H, H-1"), 7.35 (t, J 7.9 Hz, 1H, H-6), 7.50-7.64 (m, 5H, H-7, H-8, H-3', H-4', H-5'), 7.99 (d, J 7.2 Hz, 2H, H-2', H-6'), 8.18 (d, J 7.9 Hz, 1H, H-5), 8.66 (t, 1H, J 5.8 Hz, $\mathrm{CONH}$ ), 8.87 (s, H-4), 8.93 (s, 1H, NH, H-9); ${ }^{13} \mathrm{C}$ NMR (75 $\left.\mathrm{MHz}, \mathrm{CDCl}_{3}\right) \delta_{\mathrm{C}} 41.3\left(\mathrm{CH}_{2}, \mathrm{C}-1\right.$ ") $), 43.9\left(\mathrm{CH}_{2}, \mathrm{C}-2\right.$ '), 111.8 (CH, C-8), 113.6 (CH, C-4), 121.1 (CH, C-6), 122.2 (CH, C-5), 122.2 (C, C-4b), 128.2 (2CH, C-2', C-6'), 129.0 (CH, C-4'), 129.3 (CH, C-7), 129.3 (CH, C-3', C-5'), $130.6\left(\mathrm{C}_{0}, \mathrm{C}-4 \mathrm{a}\right), 134.9\left(\mathrm{C}_{0}, \mathrm{C}-9 \mathrm{a}\right), 137.8\left(\mathrm{C}_{0}, \mathrm{C}-1{ }^{\prime}\right)$, $140.0\left(\mathrm{C}_{0}, \mathrm{C}-3\right), 140.7$ (C, $\left.\mathrm{C}-1\right), 141.1\left(\mathrm{C}_{0}, \mathrm{C}-8 \mathrm{a}\right), 165.8$ $(\mathrm{C}=\mathrm{O})$; HRMS-ESI $\mathrm{m} / \mathrm{z}$, calcd. for $\mathrm{C}_{20} \mathrm{H}_{17} \mathrm{ClN}_{3} \mathrm{O}[\mathrm{M}+\mathrm{H}]^{+}$: 350.1055, found: 350.1049 . 
$N$-(2-Chloroethyl)-1-(2-fluorophenyl)- $9 H$ - $\beta$-carboline3-carboxamide (6b)

Yield: $75 \%$; mp $171-172{ }^{\circ} \mathrm{C}$; ${ }^{1} \mathrm{H}$ NMR $(300 \mathrm{MHz}$, $\left.\mathrm{CDCl}_{3}\right) \delta_{\mathrm{H}} 3.77$ (t, $J 5.8 \mathrm{~Hz}, 2 \mathrm{H}, \mathrm{H}-2$ "), 3.89 (q, $J 5.8 \mathrm{~Hz}$, 2H, H-1"), 7.29-7.44 (m, 3H, H-6, H-3', H-6'), 7.50-7.63 (m, 3H, H-4', H-7, H-8), 7.90 (td, J 7.6, 1.7 Hz, 1H, H-5'), 8.23 (d, J $7.9 \mathrm{~Hz}, 1 \mathrm{H}, \mathrm{H}-5), 8.58-8.63$ (m, 2H, CONH, $\mathrm{NH}, \mathrm{H}-9), 8.95$ (s, $1 \mathrm{H}, \mathrm{H}-4) ;{ }^{13} \mathrm{C}$ NMR $\left(75 \mathrm{MHz}, \mathrm{CDCl}_{3}\right)$ $\delta_{\mathrm{C}} 41.2\left(\mathrm{CH}_{2}, \mathrm{C}-1 "\right), 43.9\left(\mathrm{CH}_{2}, \mathrm{C}-2 "\right), 111.8(\mathrm{CH}, \mathrm{C}-8)$, 114.1 (CH, C-4), 116.3 (CH, d, J $22.5 \mathrm{~Hz}, \mathrm{C}-3$ ') 121.0 (CH, C-6), $122.0\left(\mathrm{C}_{0}, \mathrm{C}-4 \mathrm{~b}\right), 122.2(\mathrm{CH}, \mathrm{C}-5), 125.2(\mathrm{CH}$, C-6'), 125.3 ( $\left.\mathrm{C}_{0}, \mathrm{C}-4 \mathrm{a}\right), 129.1$ (CH, C-7), $130.6\left(\mathrm{C}_{0}, \mathrm{C}-1^{\prime}\right.$ '), $131.2\left(\mathrm{C}_{0}, \mathrm{~d}, J 8.4 \mathrm{~Hz}, \mathrm{C}-4\right.$ '), $132.3(\mathrm{CH}, \mathrm{d}, J 3.7 \mathrm{~Hz}$, C-5'), 135.6 (C $\left.\mathrm{C}_{0}, \mathrm{C}-9 \mathrm{a}\right), 136.3\left(\mathrm{C}_{0}, \mathrm{C}-3\right), 140.1\left(\mathrm{C}_{0}, \mathrm{C}-1\right)$, $140.6\left(\mathrm{C}_{0}, \mathrm{C}-8 \mathrm{a}\right), 159.9$ ( $\mathrm{C}_{0}$, d, J $246 \mathrm{~Hz}, 1 \mathrm{C}, \mathrm{C}-2$ '), 165.6 $(\mathrm{C}=\mathrm{O})$; HRMS-ESI $\mathrm{m} / z$, calcd. for $\mathrm{C}_{20} \mathrm{H}_{16} \mathrm{ClFN}_{3} \mathrm{O}[\mathrm{M}+\mathrm{H}]^{+}$: 368.0960, found: 368.0963 .

$\mathrm{N}$-(2-Chloroethyl)-1-(4-fluorophenyl)- $9 \mathrm{H}$ - $\beta$-carboline3-carboxamide (6c)

Yield: 91\%; mp 164-167 ${ }^{\circ} \mathrm{C}$; ${ }^{1} \mathrm{H}$ NMR $(300 \mathrm{MHz}$, $\mathrm{CDCl}_{3} / \mathrm{CD}_{3} \mathrm{OD}$ ) $\delta_{\mathrm{H}} 3.77(\mathrm{t}, J 6.6 \mathrm{~Hz}, 2 \mathrm{H}, \mathrm{H}-2$ "), $3.87(\mathrm{~m}$, 2H, H-1"), 7.27-7.36 (m, 3H, H-6, H-3', H-5'), 7.58 (m, 2H, H-7, H-8), 7.99 (dd, J 8.7, 5.3 Hz, 2H, H-2', H-6'), 8.17 (d, J 7.8 Hz, 1H, H-5), 8.67 (t, J 5.8 Hz, CONH), 8.82 (s, 1H, H-4), 9.81 (s, 1H, NH, H-9); ${ }^{13} \mathrm{C}$ NMR (75 MHz, $\left.\mathrm{CDCl}_{3} / \mathrm{CD}_{3} \mathrm{OD}\right) \delta_{\mathrm{C}} 41.2\left(\mathrm{CH}_{2}, \mathrm{C}-1 "\right), 43.8\left(\mathrm{CH}_{2}, \mathrm{C}-2 "\right)$, 112.0 (CH, C-8), 113.6 (CH, C-4), 116.1 (d, J $21.9 \mathrm{~Hz}$, 2CH, C-3', C-5'), 120.9 (CH, C-6), 122.0 (CH, C-5), 122.1 (C $\left.\mathrm{C}_{0}, \mathrm{C}-4 \mathrm{~b}\right), 128.9$ (CH, C-7), 130.2 (d, J 8.5 Hz, 2 CH, C-2', C-6'), 130.7 ( $\left.\mathrm{C}_{0}, \mathrm{C}-4 \mathrm{a}\right), 134.0\left(\mathrm{C}_{0}, \mathrm{C}-9 \mathrm{a}\right), 135.0\left(\mathrm{C}_{0}, \mathrm{C}-1^{\prime}\right)$, $139.4\left(\mathrm{C}_{0}, \mathrm{C}-3\right), 140.3\left(\mathrm{C}_{0}, \mathrm{C}-1\right), 141.2\left(\mathrm{C}_{0}, \mathrm{C}-8 \mathrm{a}\right), 163.3(\mathrm{~d}$, $J 247.7$ Hz, C ${ }_{0}, C_{-} 4$ '), 166.1 (C=O); HRMS-ESI $\mathrm{m} / z$, calcd. for $\mathrm{C}_{20} \mathrm{H}_{16} \mathrm{ClFN}_{3} \mathrm{O}[\mathrm{M}+\mathrm{H}]^{+}:$368.0960, found: 368.0957 .

$N$-(2-Chloroethyl)-1-(2-chlorophenyl)-9H- $\beta$-carboline3-carboxamide $(\mathbf{6 d})$

Yield: 94\%; mp 246-248 ${ }^{\circ} \mathrm{C}$; ${ }^{1} \mathrm{H}$ NMR $(300 \mathrm{MHz}$, $\left.\mathrm{CDCl}_{3}\right) \delta_{\mathrm{H}} 3.76(\mathrm{t}, J 5.8 \mathrm{~Hz}, 2 \mathrm{H}, \mathrm{H}-2$ "), 3.86 (q, J $5.8 \mathrm{~Hz}$, 2H, H-1"), 7.37 (t, J 7.8 Hz, 1H, H-6), 7.47-7.68 (m, 6H, H-7, H-8, H-3', H-4', H-5', H-6'), 8.23 (d, J 7.8 Hz, 1H, H-5), 8.54 (m, 2H, CONH, NH, H-9), 8.95 (s, 1H, H-4); ${ }^{13} \mathrm{C} \mathrm{NMR}\left(75 \mathrm{MHz}, \mathrm{CDCl}_{3}\right) \delta_{\mathrm{C}} 41.4\left(\mathrm{CH}_{2}, \mathrm{C}-1\right.$ "), $43.7\left(\mathrm{CH}_{2}\right.$, C-2"), 111.8 (CH, C-8), 114.2 (CH, C-4), 121.0 (CH, C-6), $122.1\left(\mathrm{C}_{0}, \mathrm{C}-4 \mathrm{~b}\right), 122.2(\mathrm{CH}, \mathrm{C}-5), 127.4(\mathrm{CH}, \mathrm{C}-7), 129.1$ (CH, C-5'), 130.2 ( $\left.\mathrm{C}_{0}, \mathrm{C}-4 \mathrm{a}\right), 130.4\left(\mathrm{CH}, \mathrm{C}-3^{\prime}\right), 130.5$ (CH, C-6'), 131.9 (CH, C-4'), $133.0\left(\mathrm{C}_{0}, \mathrm{C}-2^{\prime}\right), 135.4\left(\mathrm{C}_{0}\right.$, C-9a), 136.3 (C $\mathrm{C}_{0}, \mathrm{C}-1$ '), 139.5 ( $\left.\mathrm{C}_{0}, \mathrm{C}-3\right), 139.8$ ( $\left.\mathrm{C}_{0}, \mathrm{C}-1\right)$, $140.7\left(\mathrm{C}_{0}, \mathrm{C}-8 \mathrm{a}\right), 165.7(\mathrm{C}=\mathrm{O})$; HRMS-ESI $\mathrm{m} / \mathrm{z}$, calcd. for $\mathrm{C}_{20} \mathrm{H}_{16} \mathrm{Cl}_{2} \mathrm{~N}_{3} \mathrm{O}[\mathrm{M}+\mathrm{H}]^{+}$: 384.0665, found: 384.0665 .
$N$-(2-Chloroethyl)-1-(4-chlorophenyl)-9H- $\beta$-carboline3-carboxamide (6e)

Yield: 68\%; mp 269-272 ${ }^{\circ} \mathrm{C}$; ${ }^{1} \mathrm{H}$ NMR $(300 \mathrm{MHz}$, $\left.\mathrm{CDCl}_{3} / \mathrm{CD}_{3} \mathrm{OD}\right) \delta_{\mathrm{H}} 3.78(\mathrm{t}, J 5.8 \mathrm{~Hz}, 2 \mathrm{H}, \mathrm{H}-2 "), 3.88(\mathrm{t}$, $J 5.8 \mathrm{~Hz}, 2 \mathrm{H}, \mathrm{H}-1$ "), 7.33-7.36 (m, 1H, H-6), 7.59 (m, 4H, H-7, H-8, H-3', H-5'), 7.98 (d, J 8.1 Hz, 2H, H-2', H-6'), 8.20 (d, J 7.9 Hz, 1H, H-5), 8.83 (s, 1H, H-4); ${ }^{13} \mathrm{C}$ NMR (75 MHz, $\left.\mathrm{CDCl}_{3} / \mathrm{CD}_{3} \mathrm{OD}\right) \delta_{\mathrm{C}} 41.3\left(\mathrm{CH}_{2}, \mathrm{C}-1\right.$ ") $), 43.8\left(\mathrm{CH}_{2}\right.$, C-2"), 112.2 (CH, C-8), 113.8 (CH, C-4), 120.9 (CH, C-6), 122.0 (CH, C-5), $122.0\left(\mathrm{C}_{0}, \mathrm{C}-4 \mathrm{~b}\right), 129.0(\mathrm{CH}, \mathrm{C}-7), 129.3$ (2CH, C-3', C-5'), 129.8 (2CH, C-2', C-6'), $130.8\left(\mathrm{C}_{0}\right.$, C-4a), $135.0\left(\mathrm{C}_{0}, \mathrm{C}^{-4}\right.$ '), $135.2\left(\mathrm{C}_{0}, \mathrm{C}-9 \mathrm{a}\right), 136.4\left(\mathrm{C}_{0}, \mathrm{C}-1^{\prime}\right)$, $139.3\left(\mathrm{C}_{0}, \mathrm{C}-3\right), 140.1\left(\mathrm{C}_{0}, \mathrm{C}-1\right), 141.4\left(\mathrm{C}_{0}, \mathrm{C}-8 \mathrm{a}\right), 166.3$ $(\mathrm{C}=\mathrm{O})$; HRMS-ESI $\mathrm{m} / z$, calcd. for $\mathrm{C}_{20} \mathrm{H}_{16} \mathrm{Cl}_{2} \mathrm{~N}_{3} \mathrm{O}[\mathrm{M}+\mathrm{H}]^{+}$: 384.0665, found: 384.0673 .

$\mathrm{N}$-(2-Chloroethyl)-1-(3-nitrophenyl)- $9 H$ - $\beta$-carboline3-carboxamide (6f)

Yield: 72\%; mp 186-190 ${ }^{\circ} \mathrm{C}$; ${ }^{1} \mathrm{H}$ NMR $(300 \mathrm{MHz}$, DMSO- $\left.d_{6}\right) \delta_{\mathrm{H}} 3.70-3.84$ (m, 4H, H-1", H-2"), 7.35 (td, J 7.4, 1.1 Hz, 1H, H-6), 7.61-7.71 (m, 2H, H-7, H-8), 7.96 (t, $\left.J 8.0 \mathrm{~Hz}, 1 \mathrm{H}, \mathrm{H}-5^{\prime}\right), 8.40-8.48$ (m, 2H, H-5, H-4'), 8.59 (dt, $J$ 8.0, $1.1 \mathrm{~Hz}, 1 \mathrm{H}, \mathrm{H}-6$ '), 8.86 (t, $J 1.9 \mathrm{~Hz}, 1 \mathrm{H}, \mathrm{H}-2^{\prime}$ ), 8.93 (s, 1H, H-4), 9.01 (t, J $5.8 \mathrm{~Hz}, 1 \mathrm{H}, \mathrm{CONH}$ ), 12.06 (s, $1 \mathrm{H}, \mathrm{NH}, \mathrm{H}-9) ;{ }^{13} \mathrm{C}$ NMR $\left(75 \mathrm{MHz}\right.$, DMSO- $\left.d_{6}\right) \delta_{\mathrm{C}} 41.0$ $\left(\mathrm{CH}_{2}, \mathrm{C}-1 "\right), 43.3\left(\mathrm{CH}_{2}, \mathrm{C}-2 "\right), 112.6(\mathrm{CH}, \mathrm{C}-8), 114.1(\mathrm{CH}$, C-4), 120.5 (CH, C-6), 121.2 (C $\left.\mathrm{C}_{0}, \mathrm{C}-4 \mathrm{~b}\right), 122.3$ (CH, C-5), 123.5 (CH, C-2'), 123.6 (CH, C-4'), 129.0 (CH, C-7), 130.4 $\left(\mathrm{C}_{0}, \mathrm{C}-4 \mathrm{a}\right), 130.4\left(\mathrm{CH}, \mathrm{C}-5\right.$ ') 134.5 (CH, C-6'), $135.4\left(\mathrm{C}_{0}\right.$, C-9a), $138.3\left(\mathrm{C}_{0}, \mathrm{C}-1^{\prime}\right), 138.9\left(\mathrm{C}_{0}, \mathrm{C}-3\right), 139.7\left(\mathrm{C}_{0}, \mathrm{C}-1\right)$, $141.7\left(\mathrm{C}_{0}, \mathrm{C}-8 \mathrm{a}\right), 148.3\left(\mathrm{C}_{0}, \mathrm{C}^{\prime}{ }^{\prime}\right.$ ) $), 164.9$ (C=O); HRMSESI $m / z$, calcd. for $\mathrm{C}_{20} \mathrm{H}_{16} \mathrm{ClN}_{4} \mathrm{O}_{3}[\mathrm{M}+\mathrm{H}]^{+}$: 395.0905, found: 395.0905 .

$N$-(2-Chloroethyl)-1-(4-nitrophenyl)- $9 H$ - $\beta$-carboline3-carboxamide $(\mathbf{6 g})$

Yield: 79\%; mp > $279{ }^{\circ} \mathrm{C}$ (decomp.); ${ }^{1} \mathrm{H}$ NMR (300 MHz, DMSO- $\left.d_{6}\right) \delta_{\mathrm{H}} 3.73-3.84$ (m, 4H, H-1", H-2"), 7.35 (td, J 7.4, 1.1 Hz, 1H, H-6), 7.61-7.72 (m, 2H, H-7, H-8), 8.45-8.47 (m, 5H, H-5, H-2', H-3', H-5', H-6'), 8.94 (s, 1H, H-4), 9.01 (t, J $5.8 \mathrm{~Hz}, \mathrm{CONH}$ ), 12.07 (s, $1 \mathrm{H}, \mathrm{NH}, \mathrm{H}-9) ;{ }^{13} \mathrm{C}$ NMR $\left(75 \mathrm{MHz}, \mathrm{DMSO}-d_{6}\right) \delta_{\mathrm{C}} 41.0$ $\left(\mathrm{CH}_{2}, \mathrm{C}-1 "\right), 43.2\left(\mathrm{CH}_{2}, \mathrm{C}-2 "\right), 112.6(\mathrm{CH}, \mathrm{C}-8), 114.3$ (CH, C-4), 120.5 (CH, C-6), 121.1 (CH, C-5), 122.3 $\left(\mathrm{C}_{0}, \mathrm{C}-4 \mathrm{~b}\right), 123.8$ (2CH, C-3', C-5'), $129.1(\mathrm{CH}, \mathrm{C}-7)$, 130.1 (2CH, C-2', C-6'), 130.7 ( $\left.\mathrm{C}_{0}, \mathrm{C}-4 \mathrm{a}\right), 134.6\left(\mathrm{C}_{0}\right.$, C-9a), $138.0\left(\mathrm{C}_{0}, \mathrm{C}-1^{\prime}\right), 139.7\left(\mathrm{C}_{0}, \mathrm{C}-3\right), 141.7\left(\mathrm{C}_{0}, \mathrm{C}-1\right)$, $143.6\left(\mathrm{C}_{0}, \mathrm{C}-8 \mathrm{a}\right), 147.5\left(\mathrm{C}_{0}, \mathrm{C}-4\right.$ '), $164.8(\mathrm{C}=\mathrm{O})$; HRMSESI $m / z$, calcd. for $\mathrm{C}_{20} \mathrm{H}_{16} \mathrm{ClN}_{4} \mathrm{O}_{3}[\mathrm{M}+\mathrm{H}]^{+}:$395.0905, found: 395.0905 . 
$N$-(2-Chloroethyl)-1-(4-methoxyphenyl)- $9 H$ - $\beta$-carboline3-carboxamide (6h)

Yield: 82\%; mp 169-173 ${ }^{\circ} \mathrm{C} ;{ }^{1} \mathrm{H} \mathrm{NMR}\left(300 \mathrm{MHz}, \mathrm{CDCl}_{3}\right.$ ) $\delta_{\mathrm{H}} 3.78$ (t, J 5.8 Hz, 2H, H-2"), 3.86-3.91 (m, 2H, H-1"), $3.91\left(\mathrm{~s}, 3 \mathrm{H}, \mathrm{OCH}_{3}\right), 7.14\left(\mathrm{~d}, J 8.7 \mathrm{~Hz}, 2 \mathrm{H}, \mathrm{H}-3^{\prime}, \mathrm{H}-5\right.$ ') 7.36 (t, $J 7.9 \mathrm{~Hz}, 1 \mathrm{H}, \mathrm{H}-6), 7.55-7.62$ (m, 2H, H-7, H-8), 7.95 (d, J 8.7 Hz, 2H, H-2', H-6'), 8.20 (d, J 7.9 Hz, 1H, H-5), 8.67 (t, J $5.9 \mathrm{~Hz}, \mathrm{CONH}), 8.84$ (s, $1 \mathrm{H}, \mathrm{H}-4), 8.88$ (s, $1 \mathrm{H}$, $\mathrm{NH}, \mathrm{H}-9) ;{ }^{13} \mathrm{C}$ NMR $\left(75 \mathrm{MHz}, \mathrm{CDCl}_{3}\right) \delta_{\mathrm{C}} 41.3\left(\mathrm{CH}_{2}, \mathrm{C}-1\right.$ "), $43.9\left(\mathrm{CH}_{2}, \mathrm{C}-2\right.$ "), $55.5\left(\mathrm{OC}_{3}\right), 111.8(\mathrm{CH}, \mathrm{C}-8), 113.2$ (CH, C-4), 114.7 (2CH, C-3', C-5'), 121.0 (CH, C-6), 122.1 (CH, C-5), $122.3\left(\mathrm{C}_{0}, \mathrm{C}-4 \mathrm{~b}\right), 128.8(\mathrm{CH}, \mathrm{C}-7), 129.5(2 \mathrm{CH}$, C-2', C-6'), 130.3 ( $\left.\mathrm{C}_{0}, \mathrm{C}-4 \mathrm{a}\right), 130.4\left(\mathrm{C}_{0}, \mathrm{C}-9 \mathrm{a}\right), 134.8\left(\mathrm{C}_{0}\right.$, C-1'), $139.9\left(\mathrm{C}_{0}, \mathrm{C}-3\right), 140.7\left(\mathrm{C}_{0}, \mathrm{C}-1\right), 141.1\left(\mathrm{C}_{0}, \mathrm{C}-8 \mathrm{a}\right)$, $160.5\left(\mathrm{C}_{0}, \mathrm{C}^{4} 4^{\prime}\right), 165.9(\mathrm{C}=\mathrm{O})$; HRMS-ESI $\mathrm{m} / \mathrm{z}$, calcd. for $\mathrm{C}_{21} \mathrm{H}_{19} \mathrm{ClN}_{3} \mathrm{O}_{2}[\mathrm{M}+\mathrm{H}]^{+}: 380.1160$, found: 380.1153 .

$N$-(2-Chloroethyl)-1-[4-(dimethylamino)phenyl]$9 \mathrm{H}$ - $\beta$-carboline-3-carboxamide (6i)

Yield: $85 \%$; $\mathrm{mp}>304{ }^{\circ} \mathrm{C}$ (decomp.); ${ }^{1} \mathrm{H}$ NMR $\left(300 \mathrm{MHz}, \mathrm{CDCl}_{3}\right) \delta_{\mathrm{H}} 3.04\left(\mathrm{~s}, 6 \mathrm{H}, \mathrm{N}\left(\underline{\mathrm{CH}}_{3}\right)_{2}\right), 3.76(\mathrm{t}$, $J 6.0 \mathrm{~Hz}, 2 \mathrm{H}, \mathrm{H}-2$ "), 3.88 (q, J $6.0 \mathrm{~Hz}, 2 \mathrm{H}, \mathrm{H}-1$ "), 6.90 (d, $J 8.8 \mathrm{~Hz}, 2 \mathrm{H}, \mathrm{H}-3$ ', H-5'), 7.33 (ddd, J 7.9, $5.3,2.7 \mathrm{~Hz}, 1 \mathrm{H}$, H-6), 7.53-7.57 (m, 2H, H-7, H-8), 7.89 (d, J 8.8 Hz, 2H, H-2', H-6'), 8.18 (d, J 7.9 Hz, 1H, H-5), 8.72 (t, J 6.0 Hz, $\mathrm{CONH}$ ), 8.78 (s, 1H, H-4), 8.90 (s, 1H, NH, H-9); ${ }^{13} \mathrm{C} \mathrm{NMR}$ $\left(75 \mathrm{MHz}, \mathrm{CDCl}_{3}\right) \delta_{\mathrm{C}} 40.3\left(\mathrm{~N}\left(\mathrm{C}_{3}\right)_{2}\right), 41.3\left(\mathrm{CH}_{2}, \mathrm{C}-1 "\right), 43.9$ ( $\mathrm{CH}_{2}, \mathrm{C}-2$ "), 111.7 (CH, C-8), 112.5 (2CH, C-3', C-5'), 112.5 (CH, C-4), 120.8 (CH, C-6), $122.1(\mathrm{CH}, \mathrm{C}-5), 122.4$ $\left(\mathrm{C}_{0}, \mathrm{C}-4 \mathrm{~b}\right), 125.4\left(\mathrm{C}_{0}, \mathrm{C}-4 \mathrm{a}\right), 128.6(\mathrm{CH}, \mathrm{C}-7), 129.0(2 \mathrm{CH}$, C-2', C-6'), 130.0 ( $\left.\mathrm{C}_{0}, \mathrm{C}-9 \mathrm{a}\right), 134.7$ (C $\left.\mathrm{C}_{0}, \mathrm{C}-1^{\prime}\right), 139.8\left(\mathrm{C}_{0}\right.$, C-3), $140.6\left(\mathrm{C}_{0}, \mathrm{C}-1\right), 141.8\left(\mathrm{C}_{0}, \mathrm{C}-8 \mathrm{a}\right), 151.0\left(\mathrm{C}_{0}, \mathrm{C}-4^{\prime}\right)$, $166.1(\mathrm{C}=\mathrm{O})$; HRMS-ESI $\mathrm{m} / z$, calcd. for $\mathrm{C}_{22} \mathrm{H}_{22} \mathrm{ClN}_{4} \mathrm{O}$ $[\mathrm{M}+\mathrm{H}]^{+}:$393.1477, found: 393.1465.

\section{$\mathrm{N}$-(3-Chloropropyl)-1-phenyl-9H- $\beta$-carboline-3-carboxamide} (7a)

Yield: $82 \%$; mp 161-164 ${ }^{\circ} \mathrm{C}$; ${ }^{1} \mathrm{H}$ NMR $(500 \mathrm{MHz}$, $\left.\mathrm{CDCl}_{3}\right) \delta_{\mathrm{H}} 2.16$ (qt, $\left.J 6.4 \mathrm{~Hz}, 2 \mathrm{H}, \mathrm{H}-2 "\right), 3.65-3.70(\mathrm{~m}, 4 \mathrm{H}$, H-1", H-3"), 7.34 (t, J 7.8 Hz, 1H, H-6), 7.51-7.62 (m, 5H, H-7, H-8, H-3', H-4', H-5'), 7.97 (d, J 7.3 Hz, 2H, H-2', H-6'), 8.18 (d, J 7.8 Hz, 1H, H-5), 8.42 (s, 1H, CONH), 8.87 (s, H-4), 8.97 (s, 1H, NH, H-9); ${ }^{13} \mathrm{C}$ NMR $(125 \mathrm{MHz}$, $\left.\mathrm{CDCl}_{3}\right) \delta_{\mathrm{C}} 32.6\left(\mathrm{CH}_{2}, \mathrm{C}-2\right.$ ") $), 37.0\left(\mathrm{CH}_{2}, \mathrm{C}-1 "\right), 42.7\left(\mathrm{CH}_{2}\right.$, C-3"), 111.8 (CH, C-8), 113.5 (CH, C-4), $121.0(\mathrm{CH}, \mathrm{C}-6)$, 122.2 (CH, C-5), 122.3 (C $\left.\mathrm{C}_{0}, \mathrm{C}-4 \mathrm{~b}\right), 128.2$ (2CH, C-2', C-6'), 128.9 (CH, C-4'), 129.3 (CH, C-7), 129.3 (CH, C-3', C-5'), $130.6\left(\mathrm{C}_{0}, \mathrm{C}-4 \mathrm{a}\right), 134.8\left(\mathrm{C}_{0}, \mathrm{C}-9 \mathrm{a}\right), 137.9\left(\mathrm{C}_{0}, \mathrm{C}-1^{\prime}\right)$, $140.3\left(\mathrm{C}_{0}, \mathrm{C}-3\right), 140.7\left(\mathrm{C}_{0}, \mathrm{C}-1\right), 141.1\left(\mathrm{C}_{0}, \mathrm{C}-8 \mathrm{a}\right), 165.9$ $(\mathrm{C}=\mathrm{O})$; HRMS-ESI $\mathrm{m} / z$, calcd. for $\mathrm{C}_{21} \mathrm{H}_{19} \mathrm{ClN}_{3} \mathrm{O}[\mathrm{M}+\mathrm{H}]^{+}$: 364.1211, found: 364.1181 .
$N$-(3-Chloropropyl)-1-(2-fluorophenyl)- $9 H$ - $\beta$-carboline3-carboxamide (7b)

Yield: 52\%; mp 173-174 ${ }^{\circ} \mathrm{C}$; ${ }^{1} \mathrm{H}$ NMR $(300 \mathrm{MHz}$, $\left.\mathrm{CDCl}_{3}\right) \delta_{\mathrm{H}} 2.17$ (qt, $J 6.6 \mathrm{~Hz}, 2 \mathrm{H}, \mathrm{H}-2$ "), 3.65-3.73 (m, 4H, H-1”, H-3”), 7.28-7.43 (m, 3H, H-6, H-3', H-6'), 7.50-7.62 (m, 3H, H-4', H-7, H-8), 7.88 (td, J 7.6, $1.8 \mathrm{~Hz}$, $1 \mathrm{H}, \mathrm{H}-5$ '), 8.22 (d, J $7.9 \mathrm{~Hz}, 1 \mathrm{H}, \mathrm{H}-5), 8.36$ (t, J $6.1 \mathrm{~Hz}$, $1 \mathrm{H}, \mathrm{CONH}), 8.64$ (s, 1H, NH, H-9), 8.94 (s, 1H, H-4); ${ }^{13} \mathrm{C}$ NMR $\left(75 \mathrm{MHz}, \mathrm{CDCl}_{3}\right) \delta_{\mathrm{C}} 32.6\left(\mathrm{CH}_{2}, \mathrm{C}-2\right.$ ") $), 37.0$ $\left(\mathrm{CH}_{2}, \mathrm{C}-1\right.$ "), $42.7\left(\mathrm{CH}_{2}, \mathrm{C}-3\right.$ ") $), 111.8(\mathrm{CH}, \mathrm{C}-8), 114.0$ (CH, C-4), 116.3 (CH, d, J 21.5 Hz, C-3'), $121.0(\mathrm{CH}$, C-6), $122.0\left(\mathrm{C}_{0}, \mathrm{C}-4 \mathrm{~b}\right), 122.2$ (CH, C-5), $125.2(\mathrm{CH}, \mathrm{C}-6$ '), $125.3\left(\mathrm{C}_{0}, \mathrm{C}-4 \mathrm{a}\right), 129.1$ (CH, C-7), $130.6\left(\mathrm{C}_{0}, \mathrm{C}-1^{\prime}\right), 131.1$ (CH, d, J 7.5 Hz, C-4'), 132.3 (CH, d, J $3.8 \mathrm{~Hz}, \mathrm{C}-5$ '), $135.5\left(\mathrm{C}_{0}, \mathrm{C}-9 \mathrm{a}\right), 136.2\left(\mathrm{C}_{0}, \mathrm{C}-3\right), 140.4\left(\mathrm{C}_{0}, \mathrm{C}-1\right), 140.6$ $\left(\mathrm{C}_{0}, \mathrm{C}-8 \mathrm{a}\right), 159.9\left(\mathrm{C}_{0}, \mathrm{~d}, \mathrm{~J} 246 \mathrm{~Hz}, 1 \mathrm{C}, \mathrm{C}-2^{\prime}\right), 165.7(\mathrm{C}=\mathrm{O})$; HRMS-ESI $m / z$, calcd. for $\mathrm{C}_{21} \mathrm{H}_{18} \mathrm{ClFN}_{3} \mathrm{O}[\mathrm{M}+\mathrm{H}]^{+}$: 382.1117, found: 382.1085 .

$\mathrm{N}$-(3-Chloropropyl)-1-(4-fluorophenyl)-9H- $\beta$-carboline3-carboxamide (7c)

Yield: 92\%; mp 131-133 ${ }^{\circ} \mathrm{C}$; ${ }^{1} \mathrm{H}$ NMR $(300 \mathrm{MHz}$, $\left.\mathrm{CDCl}_{3}\right) \delta_{\mathrm{H}} 2.17$ (qt, $J 6.5 \mathrm{~Hz}, 2 \mathrm{H}, \mathrm{H}-2$ "), 3.65-3.73 (m, 4H, H-1”, H-3”), 7.28-7.39 (m, 3H, H-6, H-3', H-5'), 7.55-7.62 (m, 2H, H-7, H-8), 7.97 (dd, J 8.5, $5.3 \mathrm{~Hz}, 2 \mathrm{H}$, H-2', H-6'), 8.19 (d, J 8.0 Hz, 1H, H-5), 8.38 (t, J $6.0 \mathrm{~Hz}$, $\mathrm{CONH}), 8.83$ (s, 1H, NH, H-9), 8.87 (s, 1H, H-4); ${ }^{13} \mathrm{C} \mathrm{NMR}$ $\left(75 \mathrm{MHz}, \mathrm{CDCl}_{3}\right) \delta_{\mathrm{C}} 32.6\left(\mathrm{CH}_{2}, \mathrm{C}-2 "\right), 37.0\left(\mathrm{CH}_{2}, \mathrm{C}-1\right.$ "), $42.7\left(\mathrm{CH}_{2}, \mathrm{C}-3\right.$ ") $), 111.8$ (CH, C-8), $113.6(\mathrm{CH}, \mathrm{C}-4), 116.4$ (d, J $21.3 \mathrm{~Hz}, 2 \mathrm{CH}, \mathrm{C}^{-3}$ ', C-5'), 121.2 (CH, C-6), 122.2 (CH, C-5), $122.3\left(\mathrm{C}_{0}, \mathrm{C}-4 \mathrm{~b}\right), 129.1(\mathrm{CH}, \mathrm{C}-7), 130.1$ (d, $J 8.5 \mathrm{~Hz}, 2 \mathrm{CH}, \mathrm{C}-2$ ', C-6'), 130.8 ( $\left.\mathrm{C}_{0}, \mathrm{C}-4 \mathrm{a}\right), 134.0\left(\mathrm{C}_{0}\right.$, C-1'), 134.7 (C, C-9a), 140.1 (C $\left.\mathrm{C}_{0}, \mathrm{C}-3\right), 140.4\left(\mathrm{C}_{0}, \mathrm{C}-1\right)$, 140.7 (C, C-8a), 163.3 (d, J 247.7 Hz, C 0 , C-4'), 165.8 $(\mathrm{C}=\mathrm{O})$; HRMS-ESI $\mathrm{m} / \mathrm{z}$, calcd. for $\mathrm{C}_{21} \mathrm{H}_{18} \mathrm{ClFN}_{3} \mathrm{O}[\mathrm{M}+\mathrm{H}]^{+}$: 382.1117, found: 382.1091 .

$N$-(3-Chloropropyl)-1-(2-chlorophenyl)-9H- $\beta$-carboline3-carboxamide (7d)

Yield: 95\%; mp 195-198 ${ }^{\circ} \mathrm{C}$; ${ }^{1} \mathrm{H}$ NMR $(300 \mathrm{MHz}$, $\left.\mathrm{CDCl}_{3}\right) \delta_{\mathrm{H}} 2.14$ (qt, $J 6.5 \mathrm{~Hz}, 2 \mathrm{H}, \mathrm{H}-2$ "), $3.62-3.68$ (m, 4H, H-1", H-3"), 7.35 (t, J 7.8 Hz, 1H, H-6), 7.46-7.65 (m, 6H, H-7, H-8, H-3', H-4', H-5', H-6'), 8.21 (d, J 7.8 Hz, 1H, $\mathrm{H}-5), 8.31$ (t, J 5.5 Hz, 1H, CONH), 8.63 (s, 1H, NH, H-9), $8.92(\mathrm{~s}, 1 \mathrm{H}, \mathrm{H}-4) ;{ }^{13} \mathrm{C} \mathrm{NMR}\left(75 \mathrm{MHz}, \mathrm{CDCl}_{3}\right) \delta_{\mathrm{C}} 32.6\left(\mathrm{CH}_{2}\right.$, C-2"), $36.9\left(\mathrm{CH}_{2}, \mathrm{C}-1\right.$ "), $42.6\left(\mathrm{CH}_{2}, \mathrm{C}-3\right.$ ") $), 111.8$ (CH, C-8), 114.1 (CH, C-4), 121.0 (CH, C-6), 122.1 (C, C-4b), 122.2 (CH, C-5), 127.4 (CH, C-7), 129.1 (CH, C-5'), 130.2 ( $\mathrm{C}_{0}$, C-4a), 130.4 (CH, C-3'), 130.5 (CH, C-6'), $131.9(\mathrm{CH}$, C-4'), $133.0\left(\mathrm{C}_{0}, \mathrm{C}^{2}{ }^{\prime}\right), 135.4\left(\mathrm{C}_{0}, \mathrm{C}-9 \mathrm{a}\right), 136.3\left(\mathrm{C}_{0}, \mathrm{C}-1^{\prime}\right)$, $139.4\left(\mathrm{C}_{0}, \mathrm{C}-3\right), 140.0\left(\mathrm{C}_{0}, \mathrm{C}-1\right), 140.8\left(\mathrm{C}_{0}, \mathrm{C}-8 \mathrm{a}\right), 165.8$ 
$\left(\mathrm{C}=\mathrm{O}\right.$ ); HRMS-ESI $m / z$, calcd. for $\mathrm{C}_{21} \mathrm{H}_{18} \mathrm{Cl}_{2} \mathrm{~N}_{3} \mathrm{O}[\mathrm{M}+\mathrm{H}]^{+}$: 398.0821, found: 398.0782 .

$N$-(3-Chloropropyl)-1-(4-chlorophenyl)- $9 H$ - $\beta$-carboline3-carboxamide (7e)

Yield: 93\%; mp 175-178 ${ }^{\circ} \mathrm{C}$; ${ }^{1} \mathrm{H}$ NMR $(500 \mathrm{MHz}$, $\left.\mathrm{CDCl}_{3}\right) \delta_{\mathrm{H}} 2.17$ (qt, $J 6.4 \mathrm{~Hz}, 2 \mathrm{H}, \mathrm{H}-2$ "), 3.66-3.72 (m, 4H, H-1", H-3"), 7.36 (t, J 7.8 Hz, 1H, H-6), 7.56-7.58 (m, 4H, H-7, H-8, H-3', H-5'), 7.92 (d, J 7.8 Hz, 2H, H-2', H-6'), 8.17 (d, J 7.8 Hz, 1H, H-5), 8.37 (s, 1H, CONH), 8.86 (s, $1 \mathrm{H}, \mathrm{H}-4), 8.91$ (s, $1 \mathrm{H}, \mathrm{NH}, \mathrm{H}-9) ;{ }^{13} \mathrm{C}$ NMR $(125 \mathrm{MHz}$, $\left.\mathrm{CDCl}_{3}\right) \delta_{\mathrm{C}} 32.5\left(\mathrm{CH}_{2}, \mathrm{C}-2\right.$ "), $37.0\left(\mathrm{CH}_{2}, \mathrm{C}-1\right.$ ") $), 42.7\left(\mathrm{CH}_{2}\right.$, C-3"), 111.9 (CH, C-8), 113.7 (CH, C-4), 121.2 (CH, C-6), 122.2 (CH, C-5), 122.2 (C, $\mathrm{C}-4 \mathrm{~b}), 129.1$ (CH, C-7), 129.5 (4CH, C-3', C-5', C-2', C-6'), 130.9 (C $\left.\mathrm{C}_{0}, \mathrm{C}-4 \mathrm{a}\right), 134.7$ ( $\mathrm{C}_{0}$, C-4'), 135.3 ( $\left.\mathrm{C}_{0}, \mathrm{C}-9 \mathrm{a}\right), 136.3$ (C $\mathrm{C}_{0}, \mathrm{C}-1$ '), 139.8 (C $\left.\mathrm{C}_{0}, \mathrm{C}-3\right)$, $140.4\left(\mathrm{C}_{0}, \mathrm{C}-1\right), 140.8\left(\mathrm{C}_{0}, \mathrm{C}-8 \mathrm{a}\right), 165.7(\mathrm{C}=\mathrm{O})$; HRMSESI $m / z$, calcd. for $\mathrm{C}_{21} \mathrm{H}_{18} \mathrm{Cl}_{2} \mathrm{~N}_{3} \mathrm{O}[\mathrm{M}+\mathrm{H}]^{+}: 398.0821$, found: 398.0791 .

$N$-(3-Chloropropyl)-1-(3-nitrophenyl)- $9 H$ - $\beta$-carboline3-carboxamide (7f)

Yield: 93\%; mp 203-206 ${ }^{\circ} \mathrm{C}$; ${ }^{1} \mathrm{H}$ NMR $(300 \mathrm{MHz}$, DMSO- $\left.d_{6}\right) \delta_{\mathrm{H}} 2.07$ (qt, $J 6.5 \mathrm{~Hz}, 2 \mathrm{H}, \mathrm{H}-2$ "), 3.53 (q, $J 6.5 \mathrm{~Hz}, 2 \mathrm{H}, \mathrm{H}-1$ "), 3.73 (t, J $6.5 \mathrm{~Hz}, 2 \mathrm{H}, \mathrm{H}-3$ "), 7.34 (t, $J 7.2 \mathrm{~Hz}, 1 \mathrm{H}, \mathrm{H}-6), 7.60-7.70$ (m, 2H, H-7, H-8), 7.94 (t, J 7.9 Hz, 1H, H-5'), 8.40-8.46 (m, 2H, H-5, H-4'), 8.60 (d, J 7.9 Hz, 1H, H-6'), 8.86-8.92 (m, 3H, H-2', H-4, CONH), 12.03 (s, $1 \mathrm{H}, \mathrm{NH}, \mathrm{H}-9) ;{ }^{13} \mathrm{C}$ NMR (75 MHz, DMSO- $d_{6}$ ) $\delta_{\mathrm{C}} 32.5\left(\mathrm{CH}_{2}, \mathrm{C}-2\right.$ "), $36.7\left(\mathrm{CH}_{2}, \mathrm{C}-1\right.$ "), $43.4\left(\mathrm{CH}_{2}, \mathrm{C}-3\right.$ ") $)$, 112.5 (CH, C-8), 113.9 (CH, C-4), 120.4 (CH, C-6), 121.2 $\left(\mathrm{C}_{0}, \mathrm{C}-4 \mathrm{~b}\right), 122.2(\mathrm{CH}, \mathrm{C}-5), 123.5$ (CH, C-2'), 123.5 (CH, C-4'), 128.9 (CH, C-7), 130.3 (CH, C-5'), 130.4 $\left(\mathrm{C}_{0}, \mathrm{C}-4 \mathrm{a}\right), 134.4\left(\mathrm{C}_{0}, \mathrm{C}-9 \mathrm{a}\right), 135.4\left(\mathrm{CH}, \mathrm{C}-6^{\prime}\right), 138.2\left(\mathrm{C}_{0}\right.$, C-1'), $138.9\left(\mathrm{C}_{0}, \mathrm{C}-3\right), 140.1\left(\mathrm{C}_{0}, \mathrm{C}-1\right), 141.6\left(\mathrm{C}_{0}, \mathrm{C}-8 \mathrm{a}\right)$, $148.3\left(\mathrm{C}_{0}, \mathrm{C}^{-}{ }^{\prime}\right), 164.8(\mathrm{C}=\mathrm{O})$; HRMS-ESI $\mathrm{m} / \mathrm{z}$, calcd. for $\mathrm{C}_{21} \mathrm{H}_{18} \mathrm{ClN}_{4} \mathrm{O}_{3}[\mathrm{M}+\mathrm{H}]^{+}:$409.1062, found: 409.1032.

$\mathrm{N}$-(3-Chloropropyl)-1-(4-nitrophenyl)-9H- $\beta$-carboline3-carboxamide (7g)

Yield: 83\%; mp 233-236 ${ }^{\circ} \mathrm{C}$; ${ }^{1} \mathrm{H}$ NMR $(300 \mathrm{MHz}$, DMSO- $\left.d_{6}\right) \delta_{\mathrm{H}} 2.08$ (qt, $J 6.6 \mathrm{~Hz}, 2 \mathrm{H}, \mathrm{H}-2$ "), 3.53 (q, $J 6.6 \mathrm{~Hz}, 2 \mathrm{H}, \mathrm{H}-1$ "), 3.73 (t, $J 6.6 \mathrm{~Hz}, 2 \mathrm{H}, \mathrm{H}-3$ "), 7.34 (t, $J 7.1 \mathrm{~Hz}, 1 \mathrm{H}, \mathrm{H}-6), 7.60-7.71$ (m, 2H, H-7, H-8), 8.43-8.50 (m, 5H, H-5, H-2', H-3', H-5', H-6'), 8.88-8.91 (m, 2H, $\mathrm{H}-4, \mathrm{CONH}), 12.03$ (s, 1H, NH, H-9); ${ }^{13} \mathrm{C} \mathrm{NMR}(75 \mathrm{MHz}$, DMSO- $\left.d_{6}\right) \delta_{\mathrm{C}} 32.6\left(\mathrm{CH}_{2}, \mathrm{C}-2 "\right), 36.7\left(\mathrm{CH}_{2}, \mathrm{C}-1 "\right), 43.3$ $\left(\mathrm{CH}_{2}, \mathrm{C}-3\right.$ ") $) 112.6(\mathrm{CH}, \mathrm{C}-8), 114.1(\mathrm{CH}, \mathrm{C}-4), 120.5(\mathrm{CH}$, C-6), 121.1 ( $\left.\mathrm{C}_{0}, \mathrm{C}-4 \mathrm{~b}\right), 122.2$ (CH, C-5), 123.7 (2CH, C-3', C-5'), 129.0 ( $\mathrm{CH}, \mathrm{C}-7), 130.1$ (2CH, C-2', C-6'), 130.7 ( $\mathrm{C}_{0}$, C-4a), $134.5\left(\mathrm{C}_{0}, \mathrm{C}-9 \mathrm{a}\right), 137.9\left(\mathrm{C}_{0}, \mathrm{C}-1\right.$ ') $), 140.1\left(\mathrm{C}_{0}, \mathrm{C}-3\right)$,
141.7 (C 0 , C-1), 143.7 (C $\left.\mathrm{C}_{0}, \mathrm{C}-8 \mathrm{a}\right), 147.4$ (C $\mathrm{C}_{0}, \mathrm{C}-4$ '), 164.8 $(\mathrm{C}=\mathrm{O})$; HRMS-ESI $\mathrm{m} / z$, calcd. for $\mathrm{C}_{21} \mathrm{H}_{18} \mathrm{ClN}_{4} \mathrm{O}_{3}[\mathrm{M}+\mathrm{H}]^{+}$: 409.1062, found: 409.1040 .

$\mathrm{N}$-(3-Chloropropyl)-1-(4-methoxyphenyl)-9H- $\beta$-carboline3-carboxamide (7h)

Yield: 85\%; mp 192-194 ${ }^{\circ} \mathrm{C}$; ${ }^{1} \mathrm{H}$ NMR $(500 \mathrm{MHz}$, $\left.\mathrm{CDCl}_{3}\right) \delta_{\mathrm{H}} 2.17$ (qt, $J 6.5 \mathrm{~Hz}, 2 \mathrm{H}, \mathrm{H}-2$ "), 3.66-3.71 (m, 4H, H-1", H-3"), 3.90 (s, 3H, $\mathrm{OCH}_{3}$ ), 7.13 (d, J 8.4 Hz, 2H, H-3', H-5'), 7.35 (t, J 7.9 Hz, 1H, H-6), 7.55-7.59 (m, 2H, H-7, H-8), 7.92 (d, J 8.4 Hz, 2H, H-2', H-6'), 8.19 (d, $J 7.9 \mathrm{~Hz}, 1 \mathrm{H}, \mathrm{H}-5), 8.42$ (t, J $6.0 \mathrm{~Hz}, \mathrm{CONH}), 8.84$ (s, 1H, $\mathrm{H}-4), 8.87$ (s, $1 \mathrm{H}, \mathrm{NH}, \mathrm{H}-9)$; ${ }^{13} \mathrm{C} \mathrm{NMR}\left(125 \mathrm{MHz}, \mathrm{CDCl}_{3}\right.$ ) $\delta_{\mathrm{C}} 32.6\left(\mathrm{CH}_{2}, \mathrm{C}-2 "\right), 37.0\left(\mathrm{CH}_{2}, \mathrm{C}-1\right.$ ") $), 42.7\left(\mathrm{CH}_{2}, \mathrm{C}-3\right.$ ") $)$, $55.5\left(\mathrm{OCH}_{3}\right), 111.8(\mathrm{CH}, \mathrm{C}-8), 113.1(\mathrm{CH}, \mathrm{C}-4), 114.7$ (2CH, C-3', C-5'), 121.0 (CH, C-6), 122.2 (CH, C-5), 122.4 ( $\left.\mathrm{C}_{0}, \mathrm{C}-4 \mathrm{~b}\right), 128.8$ (CH, C-7), 129.5 (2CH, C-2', C-6'), 130.3 $\left(\mathrm{C}_{0}, \mathrm{C}-4 \mathrm{a}\right), 130.4\left(\mathrm{C}_{0}, \mathrm{C}-9 \mathrm{a}\right), 134.7\left(\mathrm{C}_{0}, \mathrm{C}-\mathbf{1}^{\prime}\right), 140.3\left(\mathrm{C}_{0}\right.$, C-3), $140.7\left(\mathrm{C}_{0}, \mathrm{C}-1\right), 141.0\left(\mathrm{C}_{0}, \mathrm{C}-8 \mathrm{a}\right), 160.5\left(\mathrm{C}_{0}, \mathrm{C}-4\right.$ '), $165.9(\mathrm{C}=\mathrm{O})$; HRMS-ESI $\mathrm{m} / \mathrm{z}$, calcd. for $\mathrm{C}_{22} \mathrm{H}_{21} \mathrm{ClN}_{3} \mathrm{O}_{2}$ $[\mathrm{M}+\mathrm{H}]^{+}:$394.1317, found: 394.1338 .

$N$-(3-Chloropropyl)-1-[4-(dimethylamino)phenyl]$9 \mathrm{H}-\beta$-carboline-3-carboxamide (7i)

Yield: $62 \%$; mp 198-202 ${ }^{\circ} \mathrm{C}$; ${ }^{1} \mathrm{H}$ NMR $(300 \mathrm{MHz}$, $\left.\mathrm{CDCl}_{3}\right) \delta_{\mathrm{H}} 2.14$ (qt, $J 6.6 \mathrm{~Hz}, 2 \mathrm{H}, \mathrm{H}-2$ "), 2.99 (s, 6H, $\left.\mathrm{N}\left(\underline{\mathrm{CH}}_{3}\right)_{2}\right), 3.63-3.70$ (m, 4H, H-1", H-3"), 6.85 (d, $J 8.8 \mathrm{~Hz}, 2 \mathrm{H}, \mathrm{H}-3^{\prime}, \mathrm{H}^{-5}$ '), 7.31 (td, J 7.9, $1.3 \mathrm{~Hz}, 1 \mathrm{H}$, H-6), 7.51-7.59 (m, 2H, H-7, H-8), 7.88 (d, J $8.8 \mathrm{~Hz}$, 2H, H-2', H-6'), 8.14 (d, J 7.9 Hz, 1H, H-5), 8.48 (t, $J 6.2 \mathrm{~Hz}, \mathrm{CONH}), 8.76$ (s, 1H, H-4), $9.16(\mathrm{~s}, 1 \mathrm{H}, \mathrm{NH}$, $\mathrm{H}-9) ;{ }^{13} \mathrm{C}$ NMR $\left(75 \mathrm{MHz}, \mathrm{CDCl}_{3}\right) \delta_{\mathrm{C}} 32.6\left(\mathrm{CH}_{2}, \mathrm{C}-2\right.$ "), $36.9\left(\mathrm{CH}_{2}, \mathrm{C}-1\right.$ ") $), 40.2\left(\mathrm{~N}\left(\underline{\mathrm{CH}}_{3}\right)_{2}\right), 42.7\left(\mathrm{CH}_{2}, \mathrm{C}-3\right.$ ") $), 111.8$ (CH, C-8), 112.3 (2CH, C-3', C-5'), $112.4(\mathrm{CH}, \mathrm{C}-4)$, 120.6 (CH, C-6), 122.0 (CH, C-5), $122.3\left(\mathrm{C}_{0}, \mathrm{C}-4 \mathrm{~b}\right)$, 125.3 ( $\left.\mathrm{C}_{0}, \mathrm{C}-4 \mathrm{a}\right), 128.4(\mathrm{CH}, \mathrm{C}-7), 129.0(2 \mathrm{CH}, \mathrm{C}-2$ ', C-6'), $130.0\left(\mathrm{C}_{0}, \mathrm{C}-9 \mathrm{a}\right), 134.6\left(\mathrm{C}_{0}, \mathrm{C}-1^{\prime}\right), 139.9\left(\mathrm{C}_{0}, \mathrm{C}-3\right)$, $140.7\left(\mathrm{C}_{0}, \mathrm{C}-1\right), 141.7\left(\mathrm{C}_{0}, \mathrm{C}-8 \mathrm{a}\right), 150.9\left(\mathrm{C}_{0}, \mathrm{C}-4\right.$ '), 166.1 $(\mathrm{C}=\mathrm{O})$; HRMS-ESI $\mathrm{m} / z$, calcd. for $\mathrm{C}_{23} \mathrm{H}_{24} \mathrm{ClN}_{4} \mathrm{O}[\mathrm{M}+\mathrm{H}]^{+}$: 407.1633, found: 407.1650 .

Synthesis of 1-(substituted-phenyl)-3-(4,5-dihydro1,3-oxazol-2-yl)-9H- $\beta$-carboline (8a-8i)

To a solution of intermediates 2 -chloroethyl- $\beta$-carboline3-carboxamides 6a-6i $(0.3 \mathrm{mmol})$ in DMF $(3 \mathrm{~mL})$, it was added 2 equivalents of potassium carbonate $(0.08 \mathrm{~g})$. The reaction mixture was refluxed until complete consumption of intermediates (14-20 h). After this time, the solution was cooled, treated with $5 \mathrm{~mL}$ of distilled water and left on ice bath for $3 \mathrm{~h}$. The solid formed was filtered under vacuum, washed with distilled water, and recrystallized 
with methanol. The derivatives 8a-8i were obtained in yields in the range of $46-93 \%$.

\section{1-Phenyl-3-(4,5-dihydro-1,3-oxazol-2-yl)-9H- $\beta$-carboline} (8a)

Yield: 85\%; mp 224-226 ${ }^{\circ} \mathrm{C}$; ${ }^{1} \mathrm{H}$ NMR $(300 \mathrm{MHz}$, $\left.\mathrm{CDCl}_{3}\right) \delta_{\mathrm{H}} 4.18(\mathrm{t}, J 9.3 \mathrm{~Hz}, 2 \mathrm{H}, \mathrm{H}-4 "), 4.59$ (t, $J 9.3 \mathrm{~Hz}$, 2H, H-5"), 7.35 (t, J 7.9 Hz, 1H, H-6), 7.44-7.61 (m, 5H, H-7, H-8, H-3', H-4', H-5'), 7.96 (d, J 7.2 Hz, 2H, H-2', H-6'), 8.18 (d, J 7.9 Hz, 1H, H-5), 8.76 (s, H-4), 8.82 (s, 1H, $\mathrm{NH}, \mathrm{H}-9) ;{ }^{13} \mathrm{C} \mathrm{NMR}\left(75 \mathrm{MHz}, \mathrm{CDCl}_{3}\right) \delta_{\mathrm{C}} 55.0\left(\mathrm{CH}_{2}, \mathrm{C}-4\right.$ "), $68.2\left(\mathrm{CH}_{2}, \mathrm{C}-5\right.$ "), 111.8 (CH, C-8), 115.1 (CH, C-4), 120.9 (CH, C-6), 122.0 (CH, C-5), $122.1\left(\mathrm{C}_{0}, \mathrm{C}-4 \mathrm{~b}\right), 128.4(2 \mathrm{CH}$, C-2', C-6'), 128.8 (CH, C-4'), 129.0 ( $\mathrm{CH}, \mathrm{C}-7), 129.1(\mathrm{CH}$, C-3', C-5'), 129.9 (C, , C-4a), $134.4\left(\mathrm{C}_{0}, \mathrm{C}-9 \mathrm{a}\right), 136.9\left(\mathrm{C}_{0}\right.$, C-3), $138.0\left(\mathrm{C}_{0}, \mathrm{C}-1^{\prime}\right), 140.6\left(\mathrm{C}_{0}, \mathrm{C}-8 \mathrm{a}\right), 142.9\left(\mathrm{C}_{0}, \mathrm{C}-1\right)$, $165.0\left(\mathrm{C}_{0}, \mathrm{C}-2 "\right)$; HRMS-ESI $\mathrm{m} / z$, calcd. for $\mathrm{C}_{20} \mathrm{H}_{16} \mathrm{~N}_{3} \mathrm{O}$ $[\mathrm{M}+\mathrm{H}]^{+}:$314.1288, found: 314.1245.

1-(2-Fluorophenyl)-3-(4,5-dihydro-1,3-oxazol-2-yl)$9 \mathrm{H}-\beta$-carboline (8b)

Yield: 46\%; mp 261-263 ${ }^{\circ} \mathrm{C}$; ${ }^{1} \mathrm{H}$ NMR $(300 \mathrm{MHz}$, $\left.\mathrm{CDCl}_{3}\right) \delta_{\mathrm{H}} 4.17$ (t, J 9.5 Hz, 2H, H-4"), 4.58 (t, J $9.5 \mathrm{~Hz}$, 2H, H-5"), 7.20-7.36 (m, 3H, H-6, H-3', H-6'), 7.42-7.60 (m, 3H, H-4', H-7, H-8), 7.87 (td, J 7.5, 1.4 Hz, 1H, H-5'), 8.17 (d, J 7.8 Hz, 1H, H-5), 8.69 (s, 1H, NH, H-9), 8.80 (s, $1 \mathrm{H}, \mathrm{H}-4) ;{ }^{13} \mathrm{C}$ NMR $\left(75 \mathrm{MHz}, \mathrm{CDCl}_{3}\right) \delta_{\mathrm{C}} 55.0\left(\mathrm{CH}_{2}\right.$, C-4"), $68.2\left(\mathrm{CH}_{2}, \mathrm{C}-5 "\right), 111.8(\mathrm{CH}, \mathrm{C}-8), 115.5(\mathrm{CH}, \mathrm{C}-4)$, 116.3 (CH, d, J 21.9 Hz, C-3'), 120.8 (CH, C-6), 121.8 ( (C), C-4b), 121.9 (CH, C-5), 125.1 (CH, C-6'), 125.3 ( $\left.\mathrm{C}_{0}, \mathrm{C}-4 \mathrm{a}\right)$, 129.0 (CH, C-7), 129.8 ( $\left.\mathrm{C}_{0}, \mathrm{C}-9 \mathrm{a}\right), 130.9$ (CH, d, J 7.9 Hz, C-4'), 132.7 (CH, C-5'), $135.2\left(\mathrm{C}_{0}, \mathrm{C}-3\right), 136.8\left(\mathrm{C}_{0}, \mathrm{C}-1^{\prime}\right)$, $138.0\left(\mathrm{C}_{0}, \mathrm{C}-8 \mathrm{a}\right), 140.6\left(\mathrm{C}_{0}, \mathrm{C}-1\right), 159.9\left(\mathrm{C}_{0}, \mathrm{~d}, \mathrm{~J} 245 \mathrm{~Hz}\right.$, 1C, C-2'), 164.9 (C $\mathrm{C}_{0}, \mathrm{C}-2$ "); HRMS-ESI $\mathrm{m} / \mathrm{z}$, calcd. for $\mathrm{C}_{20} \mathrm{H}_{15} \mathrm{FN}_{3} \mathrm{O}[\mathrm{M}+\mathrm{H}]^{+}:$332.1194, found: 332.1166 .

1-(4-Fluorophenyl)-3-(4,5-dihydro-1,3-oxazol-2-yl)$9 H-\beta$-carboline (8c)

Yield: $51 \%$; $\mathrm{mp}>246{ }^{\circ} \mathrm{C}$ (decomp.); ${ }^{1} \mathrm{H}$ NMR (300 MHz, $\left.\mathrm{CDCl}_{3}\right) \delta_{\mathrm{H}} 4.15$ (t, J $9.5 \mathrm{~Hz}, 2 \mathrm{H}, \mathrm{H}-4$ ”), 4.57 (t, $9.5 \mathrm{~Hz}, 2 \mathrm{H}, \mathrm{H}-5$ '), 7.08 (t, J $8.8 \mathrm{~Hz}, 2 \mathrm{H}, \mathrm{H}-3$ ', H-5'), 7.33 (ddd, J 7.9, 5.7, $2.3 \mathrm{~Hz}, 1 \mathrm{H}, \mathrm{H}-6$ ), 7.53-7.56 (m, $2 \mathrm{H}$, H-7, H-8), 7.84 (dd, J 8.8, 5.4 Hz, 2H, H-2', H-6'), 8.14 (d, J 7.9 Hz, 1H, H-5), 8.70 (s, 1H, H-4), 9.16 (s, 1H, NH, $\mathrm{H}-9) ;{ }^{13} \mathrm{C}$ NMR $\left(75 \mathrm{MHz}, \mathrm{CDCl}_{3}\right) \delta_{\mathrm{C}} 54.9\left(\mathrm{CH}_{2}, \mathrm{C}-4\right.$ "), $68.2\left(\mathrm{CH}_{2}, \mathrm{C}-5 "\right), 111.9(\mathrm{CH}, \mathrm{C}-8), 115.1(\mathrm{CH}, \mathrm{C}-4), 115.9$ (d, J 21.9 Hz, 2CH, C-3', C-5'), 120.9 (CH, C-6), 121.9 (CH, C-5), $122.0\left(\mathrm{C}_{0}, \mathrm{C}-4 \mathrm{~b}\right), 128.9(\mathrm{CH}, \mathrm{C}-7), 129.9\left(\mathrm{C}_{0}\right.$, C-4a), 130.2 (d, J 8.5 Hz, 2CH, C-2', C-6'), 134.0 ( $\mathrm{C}_{0}$, C-9a), 134.3 ( $\left.\mathrm{C}_{0}, \mathrm{C}-3\right), 136.6$ (C $\left.\mathrm{C}_{0}, \mathrm{C}-1^{\prime}\right), 140.8\left(\mathrm{C}_{0}, \mathrm{C}-8 \mathrm{a}\right)$, $141.9\left(\mathrm{C}_{0}, \mathrm{C}-1\right), 163.1$ (d, J 247.2 Hz, C, $\left.\mathrm{C}^{-4}{ }^{\prime}\right), 165.0\left(\mathrm{C}_{0}\right.$,
C-2"); HRMS-ESI $m / z$, calcd. for $\mathrm{C}_{20} \mathrm{H}_{15} \mathrm{FN}_{3} \mathrm{O}[\mathrm{M}+\mathrm{H}]^{+}$: 332.1194, found: 332.1149 .

1-(2-Chlorophenyl)-3-(4,5-dihydro-1,3-oxazol-2-yl)$9 \mathrm{H}$ - $\beta$-carboline (8d)

Yield: 65\%; mp 246-249 ${ }^{\circ} \mathrm{C}$; ${ }^{1} \mathrm{H}$ NMR $(500 \mathrm{MHz}$, $\left.\mathrm{CDCl}_{3} / \mathrm{CD}_{3} \mathrm{OD}\right) \delta_{\mathrm{H}} 4.14(\mathrm{t}, J 9.6 \mathrm{~Hz}, 2 \mathrm{H}, \mathrm{H}-4 "), 4.58$ (t, $J$ 9.6 Hz, 2H, H-5"), 7.32 (m, 1H, H-6), 7.38-7.45 (m, 2H, H-7, H-6'), 7.51-7.61 (m, 4H, H-8, H-3', H-4', H-5'), 8.20 (d, J 7.9 Hz, 1H, H-5), 8.80 (s, 1H, H-4); ${ }^{13} \mathrm{C} \mathrm{NMR}$ $\left(125 \mathrm{MHz}, \mathrm{CDCl}_{3} / \mathrm{CD}_{3} \mathrm{OD}\right) \delta_{\mathrm{C}} 54.5\left(\mathrm{CH}_{2}, \mathrm{C}-4\right.$ ") $) 68.4\left(\mathrm{CH}_{2}\right.$, C-5”), 112.1 (CH, C-8), 115.9 (CH, C-4), 120.7 (CH, C-6), 121.7 ( $\left.\mathrm{C}_{0}, \mathrm{C}-4 \mathrm{~b}\right), 122.0$ (CH, C-5), 127.3 (CH, C-7), 129.0 (CH, C-5'), $129.4\left(\mathrm{C}_{0}, \mathrm{C}-4 \mathrm{a}\right), 129.8(\mathrm{CH}, \mathrm{C}-3$ ') 130.4 (CH, C-6'), 132.3 (CH, C-4'), 133.4 ( $\left.\mathrm{C}_{0}, \mathrm{C}-2^{\prime}\right), 135.4\left(\mathrm{C}_{0}\right.$, C-9a), $135.6\left(\mathrm{C}_{0}, \mathrm{C}-3\right), 136.7\left(\mathrm{C}_{0}, \mathrm{C}-1^{\prime}\right), 141.2\left(\mathrm{C}_{0}, \mathrm{C}-8 \mathrm{a}\right)$, $141.4\left(\mathrm{C}_{0}, \mathrm{C}-1\right), 165.6\left(\mathrm{C}_{0}, \mathrm{C}-2\right.$ "); HRMS-ESI $\mathrm{m} / \mathrm{z}$, calcd. for $\mathrm{C}_{20} \mathrm{H}_{15} \mathrm{ClN}_{3} \mathrm{O}[\mathrm{M}+\mathrm{H}]^{+}$: 348.0898, found: 348.0854 .

1-(4-Chlorophenyl)-3-(4,5-dihydro-1,3-oxazol-2-yl)$9 H-\beta$-carboline (8e)

Yield: 50\%; mp 278-282 ${ }^{\circ} \mathrm{C}$; ${ }^{1} \mathrm{H}$ NMR $(300 \mathrm{MHz}$, $\left.\mathrm{CDCl}_{3}\right) \delta_{\mathrm{H}} 4.19(\mathrm{t}, J 9.5 \mathrm{~Hz}, 2 \mathrm{H}, \mathrm{H}-4$ "), $4.59(\mathrm{t}, J 9.5 \mathrm{~Hz}, 2 \mathrm{H}$, H-5”), 7.36 (t, J 7.9 Hz, 1H, H-6), 7.51-7.62 (m, 4H, H-7, H-8, H-3', H-5'), 7.91 (d, J 8.2 Hz, 2H, H-2', H-6'), 8.18 (d, J 7.9 Hz, 1H, H-5), 8.68 (s, 1H, NH, H-9), 8.75 (s, 1H, $\mathrm{H}-4) ;{ }^{13} \mathrm{C}$ NMR $\left(75 \mathrm{MHz}, \mathrm{CDCl}_{3}\right) \delta_{\mathrm{C}} 55.1\left(\mathrm{CH}_{2}, \mathrm{C}-4\right.$ "), 68.3 $\left(\mathrm{CH}_{2}, \mathrm{C}-5\right.$ "), 111.8 (CH, C-8), $115.3(\mathrm{CH}, \mathrm{C}-4), 121.1(\mathrm{CH}$, C-6), 122.0 (CH, C-5), 122.0 (C, , C-4b), 129.1 (CH, C-7), 129.4 (2CH, C-3', C-5'), 129.7 (2CH, C-2', C-6'), 130.2 $\left(\mathrm{C}_{0}, \mathrm{C}-4 \mathrm{a}\right), 134.3\left(\mathrm{C}_{0}, \mathrm{C}-4^{\prime}\right), 135.1\left(\mathrm{C}_{0}, \mathrm{C}-9 \mathrm{a}\right), 136.4\left(\mathrm{C}_{0}\right.$, C-3), $137.1\left(\mathrm{C}_{0}, \mathrm{C}-1^{\prime}\right), 140.6\left(\mathrm{C}_{0}, \mathrm{C}-8 \mathrm{a}\right), 141.6\left(\mathrm{C}_{0}, \mathrm{C}-1\right)$, $164.8\left(\mathrm{C}_{0}, \mathrm{C}-2 "\right)$; HRMS-ESI $\mathrm{m} / z$, calcd. for $\mathrm{C}_{20} \mathrm{H}_{15} \mathrm{ClN}_{3} \mathrm{O}$ $[\mathrm{M}+\mathrm{H}]^{+}:$348.0898, found: 348.0906.

1-(3-Nitrophenyl)-3-(4,5-dihydro-1,3-oxazol-2-yl)$9 \mathrm{H}-\beta$-carboline (8f)

Yield: $58 \%$; mp $>250{ }^{\circ} \mathrm{C}$ (decomp.); ${ }^{1} \mathrm{H}$ NMR $(500 \mathrm{MHz}$, DMSO- $\left.d_{6}\right) \delta_{\mathrm{H}} 4.06(\mathrm{t}, J 9.5 \mathrm{~Hz}, 2 \mathrm{H}, \mathrm{H}-4$ "), $4.50(\mathrm{t}, J 9.5 \mathrm{~Hz}$, 2H, H-5"), 7.34 (t, J 7.9 Hz, 1H, H-6), 7.61-7.69 (m, 2H, H-7, H-8), 7.93 (t, J 7.9 Hz, 1H, H-5'), 8.39-8.44 (m, 2H, H-5, H-4'), 8.48 (d, J 7.9 Hz, 1H, H-6'), 8.79 (t, J 1.8 Hz, 1H, $\mathrm{H}-2^{\prime}$ ), 8.87 (s, 1H, H-4), 12.01 (s, 1H, NH, H-9); ${ }^{13} \mathrm{C} \mathrm{NMR}$ $\left(125 \mathrm{MHz}, \mathrm{DMSO}-d_{6}\right) \delta_{\mathrm{C}} 54.4\left(\mathrm{CH}_{2}, \mathrm{C}-4\right.$ ") $) 67.4\left(\mathrm{CH}_{2}\right.$, C-5”), 112.5 (CH, C-8), 115.6 (CH, C-4), 120.3 (CH, C-6), 120.8 (C, C-4b), 122.1 (CH, C-5), 123.2 (CH, C-2'), 123.4 (CH, C-4'), 128.9 (CH, C-7), 129.9 (C $\left.\mathrm{C}_{0}, \mathrm{C}-4 \mathrm{a}\right), 130.3(\mathrm{CH}$, C-5'), 133.9 ( $\left.\mathrm{C}_{0}, \mathrm{C}-9 \mathrm{a}\right), 134.8$ (CH, C-6'), 135.9 ( $\left.\mathrm{C}_{0}, \mathrm{C}-3\right)$, $139.0\left(\mathrm{C}_{0}, \mathrm{C}-1^{\prime}\right), 139.0\left(\mathrm{C}_{0}, \mathrm{C}-8 \mathrm{a}\right), 141.5\left(\mathrm{C}_{0}, \mathrm{C}-1\right), 148.1$ $\left(\mathrm{C}_{0}, \mathrm{C}^{\prime} 3^{\prime}\right), 163.4\left(\mathrm{C}_{0}, \mathrm{C}-2\right.$ '); HRMS-ESI $\mathrm{m} / \mathrm{z}$, calcd. for $\mathrm{C}_{20} \mathrm{H}_{15} \mathrm{~N}_{4} \mathrm{O}_{3}[\mathrm{M}+\mathrm{H}]^{+}:$359.1139, found: 359.1098 . 
1-(4-Nitrophenyl)-3-(4,5-dihydro-1,3-oxazol-2-yl)$9 \mathrm{H}$ - $\beta$-carboline $(\mathbf{8 g})$

Yield: $74 \%$; mp $323-326{ }^{\circ} \mathrm{C}$; ${ }^{1} \mathrm{H}$ NMR $(300 \mathrm{MHz}$, DMSO- $\left.d_{6}\right) \delta_{\mathrm{H}} 4.06(\mathrm{t}, J 9.5 \mathrm{~Hz}, 2 \mathrm{H}, \mathrm{H}-4$ ") $), 4.50(\mathrm{t}, J 9.5 \mathrm{~Hz}$, 2H, H-5"), 7.34 (ddd, J 7.9, 7.0, $1.1 \mathrm{~Hz}, 1 \mathrm{H}, \mathrm{H}-6$ ), 7.63 (ddd, $J 8.2,7.0,1.1 \mathrm{~Hz}, 1 \mathrm{H}, \mathrm{H}-7), 7.69(\mathrm{~d}, J 8.2 \mathrm{~Hz}, 1 \mathrm{H}$, H-8), 8.32 (d, J 9.0 Hz, 2H, H-2', H-6'), 8.43 (d, J 7.9 Hz, 1H, H-5), 8.47 (d, J 9.0 Hz, 2H, H-3', H-5'), 8.88 (s, $1 \mathrm{H}, \mathrm{H}-4), 12.01$ (s, 1H, NH, H-9); ${ }^{13} \mathrm{C}$ NMR $(75 \mathrm{MHz}$, DMSO- $\left.d_{6}\right) \delta_{\mathrm{C}} 54.5\left(\mathrm{CH}_{2}, \mathrm{C}-4\right.$ "), $67.5\left(\mathrm{CH}_{2}, \mathrm{C}-5 "\right), 112.6$ (CH, C-8), 115.9 (CH, C-4), 120.5 (CH, C-6), $120.9\left(\mathrm{C}_{0}\right.$, C-4b), 122.2 (CH, C-5), 123.9 (2CH, C-3', C-5'), 129.0 (CH, C-7), 129.8 (2CH, C-2', C-6'), $130.1\left(\mathrm{C}_{0}, \mathrm{C}-4 \mathrm{a}\right)$, 134.1 ( $\left.\mathrm{C}_{0}, \mathrm{C}-9 \mathrm{a}\right), 136.1\left(\mathrm{C}_{0}, \mathrm{C}-3\right), 139.0\left(\mathrm{C}_{0}, \mathrm{C}-1^{\prime}\right), 141.6$ $\left(\mathrm{C}_{0}, \mathrm{C}-8 \mathrm{a}\right), 143.9\left(\mathrm{C}_{0}, \mathrm{C}-1\right), 147.4\left(\mathrm{C}_{0}, \mathrm{C}^{\prime} \mathbf{4}^{\prime}\right), 163.5\left(\mathrm{C}_{0}\right.$, C-2"); HRMS-ESI $m / z$, calcd. for $\mathrm{C}_{20} \mathrm{H}_{15} \mathrm{~N}_{4} \mathrm{O}_{3}[\mathrm{M}+\mathrm{H}]^{+}$: 359.1139, found: 359.1101 .

1-(4-Methoxyphenyl)-3-(4,5-dihydro-1,3-oxazol-2-yl)$9 \mathrm{H}$ - $\beta$-carboline (8h)

Yield: $81 \%$; mp $247-250{ }^{\circ} \mathrm{C}$; ${ }^{1} \mathrm{H}$ NMR $(500 \mathrm{MHz}$, $\left.\mathrm{CDCl}_{3}\right) \delta_{\mathrm{H}} 3.84\left(\mathrm{~s}, 3 \mathrm{H}, \mathrm{OCH}_{3}\right), 4.16(\mathrm{t}, J 9.5 \mathrm{~Hz}, 2 \mathrm{H}, \mathrm{H}-4$ "), 4.57 (t, J 9.5 Hz, 2H, H-5"), 6.98 (d, J 8.4 Hz, 2H, H-3', H-5'), 7.33 (t, J 7.8 Hz, 1H, H-6), 7.52-7.57 (m, 2H, H-7, H-8), 7.86 (d, J 8.4 Hz, 2H, H-2', H-6'), 8.15 (d, J 7.8 Hz, $1 \mathrm{H}, \mathrm{H}-5), 8.69$ (s, 1H, H-4), 8.95 (s, 1H, NH, H-9); ${ }^{13} \mathrm{C}$ NMR $\left(125 \mathrm{MHz}, \mathrm{CDCl}_{3}\right) \delta_{\mathrm{C}} 55.0\left(\mathrm{CH}_{2}, \mathrm{C}-4\right.$ ") 55.4 $\left(\mathrm{OCH}_{3}\right), 68.2\left(\mathrm{CH}_{2}, \mathrm{C}-5\right.$ ") $), 111.8(\mathrm{CH}, \mathrm{C}-8), 114.4(2 \mathrm{CH}$, C-3', C-5'), 114.6 (CH, C-4), 120.8 (CH, C-6), $121.9(\mathrm{CH}$, C-5), 122.1 ( $\left.\mathrm{C}_{0}, \mathrm{C}-4 \mathrm{~b}\right), 128.7$ (CH, C-7), 129.6 (2CH, C-2', C-6'), $130.5\left(\mathrm{C}_{0}, \mathrm{C}-4 \mathrm{a}\right), 134.3\left(\mathrm{C}_{0}, \mathrm{C}-9 \mathrm{a}\right), 136.7\left(\mathrm{C}_{0}, \mathrm{C}-3\right)$, $136.7\left(\mathrm{C}_{0}, \mathrm{C}-1^{\prime}\right), 140.7\left(\mathrm{C}_{0}, \mathrm{C}-8 \mathrm{a}\right), 142.8\left(\mathrm{C}_{0}, \mathrm{C}-1\right), 160.2$ ( $\left.\mathrm{C}_{0}, \mathrm{C}-4^{\prime}\right), 165.1$ ( $\mathrm{C}_{0}, \mathrm{C}-2$ '); HRMS-ESI $\mathrm{m} / \mathrm{z}$, calcd. for $\mathrm{C}_{21} \mathrm{H}_{18} \mathrm{~N}_{3} \mathrm{O}_{2}[\mathrm{M}+\mathrm{H}]^{+}:$344.1394, found: 344.1396 .

1-(4-(Dimethylamino)phenyl)-3-(4,5-dihydro-1,3-oxazol2-yl)-9H- $\beta$-carboline (8i)

Yield: 93\%; mp 235-238 ${ }^{\circ} \mathrm{C}$; ${ }^{1} \mathrm{H}$ NMR $(500 \mathrm{MHz}$, $\left.\mathrm{CDCl}_{3}\right) \delta_{\mathrm{H}} 2.96\left(\mathrm{~s}, 6 \mathrm{H}, \mathrm{N}\left(\underline{\mathrm{CH}}_{3}\right)_{2}\right), 4.16(\mathrm{t}, J 9.5 \mathrm{~Hz}, 2 \mathrm{H}$, H-4"), 4.56 (t, J 9.5 Hz, 2H, H-5"), 6.72 (d, J $8.7 \mathrm{~Hz}, 2 \mathrm{H}$, H-3', H-5'), 7.30 (dt, J 8.0, 4.0 Hz, 1H, H-6), 7.52-7.53 (m, 2H, H-7, H-8), 7.81 (d, J 8.7 Hz, 2H, H-2', H-6'), 8.13 (d, J $8.0 \mathrm{~Hz}, 1 \mathrm{H}, \mathrm{H}-5), 8.63$ (s, 1H, H-4), 9.03 (s, 1H, NH, $\mathrm{H}-9) ;{ }^{13} \mathrm{C} \mathrm{NMR}\left(125 \mathrm{MHz}, \mathrm{CDCl}_{3}\right) \delta_{\mathrm{C}} 40.3\left(\mathrm{~N}\left(\mathrm{C}_{3}\right)_{2}\right), 55.0$ $\left(\mathrm{CH}_{2}, \mathrm{C}-4 "\right), 68.1\left(\mathrm{CH}_{2}, \mathrm{C}-5 "\right), 111.8(\mathrm{CH}, \mathrm{C}-8), 112.3$ (2CH, C-3', C-5'), 113.9 (CH, C-4), 120.5 (CH, C-6), 121.8 $(\mathrm{CH}, \mathrm{C}-5), 122.2\left(\mathrm{C}_{0}, \mathrm{C}-4 \mathrm{~b}\right), 125.7\left(\mathrm{C}_{0}, \mathrm{C}-4 \mathrm{a}\right), 128.4(\mathrm{CH}$, C-7), 129.2 (2CH, C-2', C-6'), $129.3\left(\mathrm{C}_{0}, \mathrm{C}-9 \mathrm{a}\right), 134.3\left(\mathrm{C}_{0}\right.$, C-3), 136.5 ( $\left.\mathrm{C}_{0}, \mathrm{C}-1^{\prime}\right), 140.6\left(\mathrm{C}_{0}, \mathrm{C}-8 \mathrm{a}\right), 143.6\left(\mathrm{C}_{0}, \mathrm{C}-1\right)$, $150.8\left(\mathrm{C}_{0}, \mathrm{C}^{\prime} \mathrm{4}^{\prime}\right), 165.3$ ( $\mathrm{C}_{0}, \mathrm{C}-2$ "'); HRMS-ESI $\mathrm{m} / \mathrm{z}$, calcd. for $\mathrm{C}_{22} \mathrm{H}_{21} \mathrm{~N}_{4} \mathrm{O}[\mathrm{M}+\mathrm{H}]^{+}$: 357.1710, found: 357.1712.
Synthesis of 1-(substituted-phenyl)-3-(5,6-dihydro4H-1,3-oxazin-2-yl)- $\beta$-carboline (9a-9h)

To a solution of intermediates 3-chloropropyl$\beta$-carboline-3-carboxamides $\mathbf{7 a - 7 h}(0.3 \mathrm{mmol})$ in DMF $(3 \mathrm{~mL})$, it was added 2 equivalents of potassium carbonate $(0.08 \mathrm{~g})$. The reaction mixture was irradiated in a microwave oven at $100 \%$ power level for 5-9 min. After all the starting material was consumed (5-9 min), the reaction mixture was poured into water and the precipitate formed was filtered under vacuum, washed with water and recrystallized with acetonitrile. The derivatives $9 \mathbf{9}-\mathbf{9 h}$ were obtained in yields in the range of $45-86 \%$.

1-Phenyl-3-(5,6-dihydro-4H-1,3-oxazin-2-yl)-9H- $\beta$-carboline (9a)

Yield: 45\%; mp 231-234 ${ }^{\circ} \mathrm{C}$; ${ }^{1} \mathrm{H}$ NMR (300 MHz, $\mathrm{CDCl}_{3} / \mathrm{CD}_{3} \mathrm{OD}$ ) $\delta_{\mathrm{H}} 2.04$ (qt, $\left.J 5.4 \mathrm{~Hz}, 2 \mathrm{H}, \mathrm{H}-5 "\right), 3.68$ (t, $J 5.4 \mathrm{~Hz}, 2 \mathrm{H}, \mathrm{H}-4$ "), 4.47 (t, $J 5.4 \mathrm{~Hz}, 2 \mathrm{H}, \mathrm{H}-6$ "), 7.257.31 (m, 1H, H-6), 7.36-7.54 (m, 5H, H-7, H-8, H-3', H-4', H-5'), 7.91 (d, J 7.0 Hz, 2H, H-2', H-6'), 8.14 (d, $J 7.8 \mathrm{~Hz}, 1 \mathrm{H}, \mathrm{H}-5), 8.61$ (s, 1H, H-4); ${ }^{13} \mathrm{C}$ NMR $(75 \mathrm{MHz}$, $\left.\mathrm{CDCl}_{3} / \mathrm{CD}_{3} \mathrm{OD}\right) \delta_{\mathrm{C}} 21.9\left(\mathrm{CH}_{2}, \mathrm{C}-5 "\right), 42.8\left(\mathrm{CH}_{2}, \mathrm{C}-4\right.$ "), $65.6\left(\mathrm{CH}_{2}, \mathrm{C}-6\right.$ ") $), 111.8(\mathrm{CH}, \mathrm{C}-8), 113.4(\mathrm{CH}, \mathrm{C}-4), 120.4$ (CH, C-6), $121.9(\mathrm{CH}, \mathrm{C}-5), 122.1\left(\mathrm{C}_{0}, \mathrm{C}-4 \mathrm{~b}\right), 128.5(2 \mathrm{CH}$, C-2', C-6'), 128.5 (CH, C-4'), 128.8 (CH, C-7), $128.9(\mathrm{CH}$, C-3', C-5'), 130.0 ( $\left.\mathrm{C}_{0}, \mathrm{C}-4 \mathrm{a}\right), 134.3\left(\mathrm{C}_{0}, \mathrm{C}-9 \mathrm{a}\right), 138.3\left(\mathrm{C}_{0}\right.$, C-1'), 140.9 (C $\left.\mathrm{C}_{0}, \mathrm{C}-8 \mathrm{a}\right), 141.6\left(\mathrm{C}_{0}, \mathrm{C}-3\right), 142.3\left(\mathrm{C}_{0}, \mathrm{C}-1\right)$, $156.3\left(\mathrm{C}_{0}, \mathrm{C}-2\right.$ "); HRMS-ESI $\mathrm{m} / z$, calcd. for $\mathrm{C}_{21} \mathrm{H}_{18} \mathrm{~N}_{3} \mathrm{O}$ $[\mathrm{M}+\mathrm{H}]^{+}:$328.1444, found: 328.1476 .

1-(2-Fluorophenyl)-3-(5,6-dihydro-4H-1,3-oxazin-2-yl)$9 H-\beta$-carboline (9b)

Yield: 73\%; mp 234-239 ${ }^{\circ} \mathrm{C}$; ${ }^{1} \mathrm{H}$ NMR $(300 \mathrm{MHz}$, $\left.\mathrm{CDCl}_{3}\right) \delta_{\mathrm{H}} 2.07$ (qt, $J 5.6 \mathrm{~Hz}, 2 \mathrm{H}, \mathrm{H}-5$ "), 3.74 (t, J $5.6 \mathrm{~Hz}$, 2H, H-4"), 4.51 (t, J 5.6 Hz, 2H, H-6"), 7.21-7.35 (m, 3H, H-6, H-3', H-6'), 7.43-7.59 (m, 3H, H-4', H-7, H-8), 7.91 (td, $J$ 7.5, 1.6 Hz, 1H, H-5'), 8.19 (d, J 7.8 Hz, 1H, H-5), 8.46 (s, 1H, NH, H-9), 8.74 (s, 1H, H-4); ${ }^{13} \mathrm{C}$ NMR $\left(75 \mathrm{MHz}, \mathrm{CDCl}_{3}\right) \delta_{\mathrm{C}} 22.0\left(\mathrm{CH}_{2}, \mathrm{C}-5\right.$ "), $43.0\left(\mathrm{CH}_{2}, \mathrm{C}-4\right.$ "), $65.7\left(\mathrm{CH}_{2}, \mathrm{C}-6 "\right), 111.7(\mathrm{CH}, \mathrm{C}-8), 113.9(\mathrm{CH}, \mathrm{C}-4), 115.8$ (CH, d, J 21.9 Hz, C-3'), 120.6 (CH, C-6), 122.0 (CH, C-5), $122.1\left(\mathrm{C}_{0}, \mathrm{C}-4 \mathrm{~b}\right), 125.1$ (CH, C-6'), $125.2\left(\mathrm{C}_{0}, \mathrm{C}-4 \mathrm{a}\right), 128.8$ (CH, C-7), 130.1 ( $\left.\mathrm{C}_{0}, \mathrm{C}-9 \mathrm{a}\right), 130.7$ (CH, d, J $8.5 \mathrm{~Hz}, \mathrm{C}-4$ '), 132.9 (CH, d, J 4.0 Hz, C-5'), 135.0 ( $\left.\mathrm{C}_{0}, \mathrm{C}^{\prime} \mathbf{1}^{\prime}\right), 137.2\left(\mathrm{C}_{0}\right.$, C-8a), 140.7 ( $\left.\mathrm{C}_{0}, \mathrm{C}-3\right), 142.2\left(\mathrm{C}_{0}, \mathrm{C}-1\right), 155.7$ (C $\mathrm{C}_{0}, \mathrm{C}-2$ "), $160.0\left(\mathrm{C}_{0}, \mathrm{~d}, \mathrm{~J} 244.9 \mathrm{~Hz}, 1 \mathrm{C}, \mathrm{C}-2\right.$ ') ; HRMS-ESI $\mathrm{m} / z$, calcd. for $\mathrm{C}_{21} \mathrm{H}_{17} \mathrm{FN}_{3} \mathrm{O}[\mathrm{M}+\mathrm{H}]^{+}$: 346.1350, found: 346.1345 .

1-(4-Fluorophenyl)-3-(5,6-dihydro-4H-1,3-oxazin-2-yl)$9 \mathrm{H}-\beta$-carboline $(9 \mathrm{c})$

Yield: 45\%; mp 228-231 ${ }^{\circ} \mathrm{C}$; ${ }^{1} \mathrm{H}$ NMR (300 MHz, 
$\left.\mathrm{CDCl}_{3} / \mathrm{CD}_{3} \mathrm{OD}\right) \delta_{\mathrm{H}} 2.06$ (dt, $J 5.7 \mathrm{~Hz}, 2 \mathrm{H}, \mathrm{H}-5$ "), 3.70 (t, $J 5.7 \mathrm{~Hz}, 2 \mathrm{H}, \mathrm{H}-4$ "), 4.49 (t, J $5.7 \mathrm{~Hz}, 2 \mathrm{H}, \mathrm{H}-6$ "), 7.14 (t, $J$ 8.7 Hz, 2H, H-3', H-5'), 7.28-7.33 (m, 1H, H-6), 7.497.57 (m, 2H, H-7, H-8), 7.91 (dd, J 8.7, 5.5 Hz, 2H, H-2', H-6'), 8.15 (d, J 7.9 Hz, 1H, H-5), 8.57 (s, 1H, H-4); ${ }^{13} \mathrm{C}$ NMR $\left(75 \mathrm{MHz}, \mathrm{CDCl}_{3} / \mathrm{CD}_{3} \mathrm{OD}\right) \delta_{\mathrm{C}} 21.9\left(\mathrm{CH}_{2}, \mathrm{C}-5 "\right)$, $42.7\left(\mathrm{CH}_{2}, \mathrm{C}-4\right.$ "), $65.6\left(\mathrm{CH}_{2}, \mathrm{C}-6 "\right), 111.9(\mathrm{CH}, \mathrm{C}-8)$, 113.4 (CH, C-4), 115.8 (d, J 21.3 Hz, 2CH, C-3', C-5'), 120.5 (CH, C-6), 121.8 (CH, C-5), 122.1 ( $\left.\mathrm{C}_{0}, \mathrm{C}-4 \mathrm{~b}\right), 128.6$ (CH, C-7), $130.1\left(\mathrm{C}_{0}, \mathrm{C}-4 \mathrm{a}\right), 130.4$ (d, J 8.5 Hz, 2CH, C-2', C-6'), $134.1\left(\mathrm{C}_{0}, \mathrm{C}-9 \mathrm{a}\right), 134.4\left(\mathrm{C}_{0}, \mathrm{C}-1^{\prime}\right), 141.0\left(\mathrm{C}_{0}\right.$, C-8a), 141.2 (C $\left.\mathrm{C}_{0}, \mathrm{C}-3\right), 141.4\left(\mathrm{C}_{0}, \mathrm{C}-1\right), 156.4\left(\mathrm{C}_{0}, \mathrm{C}-2\right.$ "), 163.1 (d, J $246.6 \mathrm{~Hz}, \mathrm{C}_{0}, \mathrm{C}-4^{\prime}$ ); HRMS-ESI $\mathrm{m} / \mathrm{z}$, calcd. for $\mathrm{C}_{21} \mathrm{H}_{17} \mathrm{FN}_{3} \mathrm{O}[\mathrm{M}+\mathrm{H}]^{+}:$346.1350, found: 346.1386.

1-(2-Chlorophenyl)-3-(5,6-dihydro-4H-1,3-oxazin-2-yl)$9 H-\beta$-carboline (9d)

Yield: 86\%; mp 236-238 ${ }^{\circ} \mathrm{C}$; ${ }^{1} \mathrm{H}$ NMR $(300 \mathrm{MHz}$, DMSO- $\left.d_{6}\right) \delta_{\mathrm{H}} 1.93$ (qt, $J 5.5 \mathrm{~Hz}, 2 \mathrm{H}, \mathrm{H}-5$ "), 3.55 (t, $J 5.5 \mathrm{~Hz}, 2 \mathrm{H}, \mathrm{H}-4$ "), 4.36 (t, J $5.5 \mathrm{~Hz}, 2 \mathrm{H}, \mathrm{H}-6$ "), 7.27 (dt, J 7.8, 4.0 Hz, 1H, H-6), 7.51-7.70 (m, 6H, H-7, H-8, H-3', H-4', H-5', H-6'), 8.35 (d, J 7.8 Hz, 1H, H-5), 8.75 (s, 1H, H-4), 11.48 (s, 1H, NH, H-9); ${ }^{13} \mathrm{C}$ NMR (75 MHz, DMSO- $\left.d_{6}\right) \delta_{\mathrm{C}} 21.6\left(\mathrm{CH}_{2}, \mathrm{C}-5 "\right), 42.1\left(\mathrm{CH}_{2}, \mathrm{C}-4\right.$ ") $), 64.7$ $\left(\mathrm{CH}_{2}, \mathrm{C}-6\right.$ "), 112.2 (CH, C-8), 113.6 (CH, C-4), 119.8 $(\mathrm{CH}, \mathrm{C}-6), 121.1\left(\mathrm{C}_{0}, \mathrm{C}-4 \mathrm{~b}\right), 122.0(\mathrm{CH}, \mathrm{C}-5), 127.4$ $(\mathrm{CH}, \mathrm{C}-7), 128.5\left(\mathrm{CH}, \mathrm{C}-5\right.$ '), $128.6\left(\mathrm{C}_{0}, \mathrm{C}-4 \mathrm{a}\right), 129.6$ (CH, C-3'), 130.4 (CH, C-6'), 132.0 (CH, C-4'), 132.6 $\left(\mathrm{C}_{0}, \mathrm{C}^{2}{ }^{\prime}\right), 134.5\left(\mathrm{C}_{0}, \mathrm{C}-9 \mathrm{a}\right), 136.9\left(\mathrm{C}_{0}, \mathrm{C}^{\prime}{ }^{\prime}{ }^{\prime}\right), 140.0$ $\left(\mathrm{C}_{0}, \mathrm{C}-8 \mathrm{a}\right), 140.7\left(\mathrm{C}_{0}, \mathrm{C}-3\right), 141.4\left(\mathrm{C}_{0}, \mathrm{C}-1\right), 154.7\left(\mathrm{C}_{0}\right.$, C-2"); HRMS-ESI $m / z$, calcd. for $\mathrm{C}_{21} \mathrm{H}_{17} \mathrm{ClN}_{3} \mathrm{O}[\mathrm{M}+\mathrm{H}]^{+}$: 362.1055, found: 362.1036 .

1-(4-Chlorophenyl)-3-(5,6-dihydro-4H-1,3-oxazine)$9 H-\beta$-carboline (9e)

Yield: 60\%; mp 234-236 ${ }^{\circ} \mathrm{C}$; ${ }^{1} \mathrm{H}$ NMR $(300 \mathrm{MHz}$, DMSO- $\left.d_{6}\right) \delta_{\mathrm{H}} 1.95$ (qt, $J 5.4 \mathrm{~Hz}, 2 \mathrm{H}, \mathrm{H}-5$ "), 3.57 (t, $J 5.4 \mathrm{~Hz}, 2 \mathrm{H}, \mathrm{H}-4$ "), 4.40 (t, J $5.4 \mathrm{~Hz}, 2 \mathrm{H}, \mathrm{H}-6$ "), 7.28 (t, J 7.9 Hz, 1H, H-6), 7.55-7.63 (m, 2H, H-7, H-8), 7.68 (d, $\left.J 8.4 \mathrm{~Hz}, 2 \mathrm{H}, \mathrm{H}-3^{\prime}, \mathrm{H}_{-} 5^{\prime}\right), 8.04$ (d, J $8.4 \mathrm{~Hz}, 2 \mathrm{H}, \mathrm{H}-2^{\prime}$, H-6'), 8.35 (d, J 7.9 Hz, 1H, H-5), 8.70 (s, 1H, H-4), 11.69 (s, $1 \mathrm{H}, \mathrm{NH}, \mathrm{H}-9) ;{ }^{13} \mathrm{C}$ NMR $\left(75 \mathrm{MHz}, \mathrm{DMSO}-d_{6}\right) \delta_{\mathrm{C}} 21.6$ ( $\mathrm{CH}_{2}, \mathrm{C}-5$ "), $42.1\left(\mathrm{CH}_{2}, \mathrm{C}-4\right.$ "), $64.8\left(\mathrm{CH}_{2}, \mathrm{C}-6\right.$ "), 112.5 (CH, C-8), 113.4 (CH, C-4), 120.0 (CH, C-6), $121.2(\mathrm{CH}$, C-4b), $121.9\left(\mathrm{C}_{0}, \mathrm{C}-5\right), 128.5(\mathrm{CH}, \mathrm{C}-7), 128.7$ (2CH, C-3', C-5'), 129.8 ( $\left.\mathrm{C}_{0}, \mathrm{C}-4 \mathrm{a}\right), 130.3$ (2CH, C-2', C-6'), $133.4\left(\mathrm{C}_{0}, \mathrm{C}^{\prime} 4^{\prime}\right), 133.5\left(\mathrm{C}_{0}, \mathrm{C}-9 \mathrm{a}\right), 136.8$ ( $\left.\mathrm{C}_{0}, \mathrm{C}^{\prime} \mathbf{1}^{\prime}\right), 139.4$ $\left(\mathrm{C}_{0}, \mathrm{C}-8 \mathrm{a}\right), 141.2\left(\mathrm{C}_{0}, \mathrm{C}-3\right), 141.6\left(\mathrm{C}_{0}, \mathrm{C}-1\right), 154.7\left(\mathrm{C}_{0}\right.$, C-2"); HRMS-ESI $m / z$, calcd. for $\mathrm{C}_{21} \mathrm{H}_{17} \mathrm{ClN}_{3} \mathrm{O}[\mathrm{M}+\mathrm{H}]^{+}$: 362.1055, found: 362.1046 .
1-(3-Nitrophenyl)-3-(5,6-dihydro-4H-1,3-oxazin-2-yl)$9 \mathrm{H}-\beta$-carboline (9f)

Yield: $51 \%$; mp 255-258 ${ }^{\circ} \mathrm{C}$; ${ }^{1} \mathrm{H}$ NMR $(300 \mathrm{MHz}$, DMSO- $\left.d_{6}\right) \delta_{\mathrm{H}} 1.96$ (qt, $J 5.5 \mathrm{~Hz}, 2 \mathrm{H}, \mathrm{H}-5$ "), 3.59 (t, $J 5.5 \mathrm{~Hz}, 2 \mathrm{H}, \mathrm{H}-4$ "), 4.42 (t, J $5.5 \mathrm{~Hz}, 2 \mathrm{H}, \mathrm{H}-6$ "), 7.31 (t, $J 7.2 \mathrm{~Hz}, 1 \mathrm{H}, \mathrm{H}-6), 7.57-7.67$ (m, 2H, H-7, H-8), 7.91 (t, J 7.6 Hz, 1H, H-5'), 8.37-8.40 (m, 2H, H-4', H-5), 8.45 (d, $J 7.6$ Hz, 1H, H-6'), 8.76-8.77 (m, 2H, H-2', H-4), 11.86 (s, $1 \mathrm{H}, \mathrm{NH}, \mathrm{H}-9) ;{ }^{13} \mathrm{C}$ NMR $\left(75 \mathrm{MHz}\right.$, DMSO- $\left.d_{6}\right) \delta_{\mathrm{C}} 21.6\left(\mathrm{CH}_{2}\right.$, C-5"), $42.1\left(\mathrm{CH}_{2}, \mathrm{C}-4\right.$ "), $64.9\left(\mathrm{CH}_{2}, \mathrm{C}-6\right.$ "), 112.5 (CH, C-8), 114.0 (CH, C-4), 120.3 (CH, C-6), 121.2 (C, C-4b), 122.1 (CH, C-5), 123.2 (CH, C-2'), 123.3 (CH, C-4'), $128.8(\mathrm{CH}$, C-7), $130.2\left(\mathrm{C}_{0}, \mathrm{C}-4 \mathrm{a}\right), 130.4\left(\mathrm{CH}, \mathrm{C}-5\right.$ ') 133.8 ( $\left.\mathrm{C}_{0}, \mathrm{C}-9 \mathrm{a}\right)$, 135.0 (CH, C-6'), $138.3\left(\mathrm{C}_{0}, \mathrm{C}-1^{\prime}\right), 139.5$ ( $\left.\mathrm{C}_{0}, \mathrm{C}-8 \mathrm{a}\right), 141.4$ $\left(\mathrm{C}_{0}, \mathrm{C}-3\right), 141.7\left(\mathrm{C}_{0}, \mathrm{C}-1\right), 148.2\left(\mathrm{C}_{0}, \mathrm{C}-3{ }^{\prime}\right), 154.6\left(\mathrm{C}_{0}\right.$, C-2"); HRMS-ESI $m / z$, calcd. for $\mathrm{C}_{21} \mathrm{H}_{17} \mathrm{~N}_{4} \mathrm{O}_{3}[\mathrm{M}+\mathrm{H}]^{+}$: 373.1295, found: 373.1295 .

1-(4-Nitrophenyl)-3-(5,6-dihydro-4H-1,3-oxazin-2-yl)$9 \mathrm{H}-\beta$-carboline $(\mathbf{9 g})$

Yield: 51\%; mp 297-300 ${ }^{\circ} \mathrm{C}$; ${ }^{1} \mathrm{H}$ NMR (300 MHz, DMSO- $\left.d_{6}\right) \delta_{\mathrm{H}} 1.96$ (qt, $J 5.5 \mathrm{~Hz}, 2 \mathrm{H}, \mathrm{H}-5$ "), 3.59 (t, $J 5.5 \mathrm{~Hz}, 2 \mathrm{H}, \mathrm{H}-4$ "), 4.41 (t, J $5.5 \mathrm{~Hz}, 2 \mathrm{H}, \mathrm{H}-6$ "), 7.31 (t, $J 7.8 \mathrm{~Hz}, 1 \mathrm{H}, \mathrm{H}-6$ ), 7.58-7.68 (m, 2H, H-7, H-8), 8.30 (d, $J 9.0 \mathrm{~Hz}, 2 \mathrm{H}, \mathrm{H}-3$ ' , H-5'), 8.39 (d, J 7.8 Hz, 1H, H-5), 8.46 (d, J $9.0 \mathrm{~Hz}, 2 \mathrm{H}, \mathrm{H}-2^{\prime}, \mathrm{H}^{-6}$ '), 8.79 (s, 1H, H-4), 11.87 (s, $1 \mathrm{H}, \mathrm{NH}, \mathrm{H}-9) ;{ }^{13} \mathrm{C}$ NMR $\left(75 \mathrm{MHz}, \mathrm{DMSO}-d_{6}\right) \delta_{\mathrm{C}} 21.6$ $\left(\mathrm{CH}_{2}, \mathrm{C}-5 "\right), 42.1\left(\mathrm{CH}_{2}, \mathrm{C}-4 "\right), 64.9\left(\mathrm{CH}_{2}, \mathrm{C}-6 "\right), 112.5$ (CH, C-8), 114.3 (CH, C-4), 120.3 (CH, C-6), $121.1\left(\mathrm{C}_{0}\right.$, C-4b), 122.0 (CH, C-5), 123.9 (2CH, C-2', C-6'), 128.9 (CH, C-7), 129.8 (2CH, C-3', C-5'), 130.3 (C, C-4a), $133.9\left(\mathrm{C}_{0}, \mathrm{C}-9 \mathrm{a}\right), 138.1\left(\mathrm{C}_{0}, \mathrm{C}-1\right.$ ' $), 141.4\left(\mathrm{C}_{0}, \mathrm{C}-8 \mathrm{a}\right), 141.7$ $\left(\mathrm{C}_{0}, \mathrm{C}-3\right), 144.3\left(\mathrm{C}_{0}, \mathrm{C}-1\right), 147.3\left(\mathrm{C}_{0}, \mathrm{C}^{\prime} \mathbf{4}^{\prime}\right)$; HRMS-ESI $\mathrm{m} / z$, calcd. for $\mathrm{C}_{21} \mathrm{H}_{17} \mathrm{~N}_{4} \mathrm{O}_{3}[\mathrm{M}+\mathrm{H}]^{+}: 373.1295$, found: 373.1281 .

1-(4-Methoxyphenyl)-3-(5,6-dihydro-4H-1,3-oxazin-2-yl)$9 \mathrm{H}-\beta$-carboline (9h)

Yield: 66\%; mp 219-221 ${ }^{\circ} \mathrm{C}$; ${ }^{1} \mathrm{H}$ NMR $(500 \mathrm{MHz}$, $\left.\mathrm{CDCl}_{3}\right) \delta_{\mathrm{H}} 2.05$ (qt, $J 5.6 \mathrm{~Hz}, 2 \mathrm{H}, \mathrm{H}-5$ "), $3.71(\mathrm{t}, J 5.6 \mathrm{~Hz}$, 2H, H-4"), 3.83 (s, 3H, $\underline{\mathrm{OCH}}_{3}$ ), 4.48 (t, J 5.6 Hz, 2H, H-6"), 6.96 (d, J 8.2 Hz, 2H, H-3', H-5'), 7.30 (t, J 7.8 Hz, 1H, H-6), 7.49-7.54 (m, 2H, H-7, H-8), 7.85 (d, J $8.2 \mathrm{~Hz}, 2 \mathrm{H}$, H-2', H-6'), 8.15 (d, J 7.8 Hz, 1H, H-5), 8.63 (s, 1H, H-4), 8.89 (s, $1 \mathrm{H}, \mathrm{NH}, \mathrm{H}-9) ;{ }^{13} \mathrm{C} \mathrm{NMR}\left(125 \mathrm{MHz}, \mathrm{CDCl}_{3}\right) \delta_{\mathrm{C}} 22.0$ $\left(\mathrm{CH}_{2}, \mathrm{C}-5\right.$ "), $42.9\left(\mathrm{CH}_{2}, \mathrm{C}-4\right.$ "), $55.4\left(\mathrm{OCH}_{3}\right), 65.6\left(\mathrm{CH}_{2}\right.$, C-6"), $111.7(\mathrm{CH}, \mathrm{C}-8), 112.9(\mathrm{CH}, \mathrm{C}-4), 114.3(2 \mathrm{CH}$, C-3', C-5'), 120.5 (CH, C-6), 121.9 (CH, C-5), $122.3\left(\mathrm{C}_{0}\right.$, C-4b), 128.4 (CH, C-7), 129.7 (2CH, C-2', C-6'), 129.9 $\left(\mathrm{C}_{0}, \mathrm{C}-4 \mathrm{a}\right), 130.9\left(\mathrm{C}_{0}, \mathrm{C}-9 \mathrm{a}\right), 134.1\left(\mathrm{C}_{0}, \mathrm{C}-3\right), 140.7\left(\mathrm{C}_{0}\right.$, C-1'), 141.9 (C $\left.\mathrm{C}_{0}, \mathrm{C}-8 \mathrm{a}\right), 142.1$ ( $\left.\mathrm{C}_{0}, \mathrm{C}-1\right), 156.0\left(\mathrm{C}_{0}, \mathrm{C}^{\prime}{ }^{\prime}\right)$, 
$160.1\left(\mathrm{C}_{0}, \mathrm{C}-2\right.$ "); HRMS-ESI $\mathrm{m} / \mathrm{z}$, calcd. for $\mathrm{C}_{22} \mathrm{H}_{20} \mathrm{~N}_{3} \mathrm{O}_{2}$ $[\mathrm{M}+\mathrm{H}]^{+}:$358.1550, found: 358.1567 .

\section{Antileishmanial activity}

Parasites and cell culture

Leishmania amazonensis promastigotes were maintained at $25^{\circ} \mathrm{C}$ in Warren medium supplemented with $10 \%$ FBS (fetal bovine serum). J774-A1 macrophages were maintained at $37^{\circ} \mathrm{C}$ under $5 \% \mathrm{CO}_{2}$ atmosphere in Roswell Park Memorial Institute (RPMI) 1640 medium (pH 7.2) supplemented with $10 \%$ FBS.

\section{Antiprotozoal activity}

The effects of synthesized compounds were evaluated in promastigotes of L. amazonensis in $\log$ phase of growth $(48 \mathrm{~h})$ at concentration of $1 \times 10^{-6}$ cells $\mathrm{mL}^{-1}$. The promastigotes were added into sterile 96 -well microplates containing increasing concentrations of compounds 6a-6i and 7a-7i, 8a-8i and 9a-9h. After incubation for $72 \mathrm{~h}$ at $25{ }^{\circ} \mathrm{C}$, it was added $50 \mu \mathrm{L}$ of solution of 2,3-bis(2-methoxy-4-nitro-5-sulfophenyl)5-[(phenylamino)carbonyl]-2H-tetrazolium hydroxide/ phenazine methosulfate (XTT/PMS) (XTT/PMS, 0.5 and $0.3 \mathrm{mg} \mathrm{mL}^{-1}$ ) in the absence of light. After $4 \mathrm{~h}$, the absorbance was read in a spectrophotometer at $450 \mathrm{~nm}$. The $\mathrm{IC}_{50}$ values (concentration that inhibit $50 \%$ of cells) was determined by linear regression. Miltefosine was used as positive control.

The effects of compounds $6 \mathbf{d}, \mathbf{8 d}, \mathbf{8 i}, 9 \mathbf{a}, 9 \mathbf{e}$ and $\mathbf{9 h}$ were also evaluated in intracellular amastigotes, in this antiproliferative assay, J774A1 macrophages $\left(5 \times 10^{5}\right.$ cells $\left.\mathrm{mL}^{-1}\right)$ and promastigotes $\left(5 \times 10^{6}\right.$ parasites $\left.\mathrm{mL}^{-1}\right)$ were added in a plate with coverslips and incubated at $34{ }^{\circ} \mathrm{C}$ with $5 \% \mathrm{CO}_{2}$ during $24 \mathrm{~h}$. The treatment was performed after $24 \mathrm{~h}$ with compounds in increasing concentrations and incubated for $48 \mathrm{~h}$. For the determination of $\mathrm{IC}_{50}$, the glass coverslips were fixed and stained with Panótico kit as indicated by the manufacturer and 200 macrophages per coverslip were evaluated on a light microscope. The number of macrophages infected, the number of amastigotes within each infected macrophage and the survival index (infected cells percentage $\times$ amastigote average per infected macrophage) were determined. Survival index of amastigotes from untreated infected macrophages was considered as $100 \%$ of survival.

\section{Cytotoxicity assay}

The cytotoxicity was evaluated in J774-A1 macrophages. The macrophages at concentration of $5 \times 10^{-5}$ cells $\mathrm{mL}^{-1}$ in RPMI 1640 medium supplemented with 10\% FBS were introduced into sterile 96-well micro plates and incubated for $24 \mathrm{~h}$ at $37{ }^{\circ} \mathrm{C}$ and $5 \%$ of $\mathrm{CO}_{2}$ tension. After this period, the supernatant was removed and increasing concentrations of the substances were added. After $48 \mathrm{~h}$ of incubation under the same conditions mentioned above, the cells were washed with $0.01 \mathrm{M}$ PBS (phosphatebuffered saline) and $50 \mu \mathrm{L}$ of 3-(4,5-dimethyl-2-thiazolyl)2,5-diphenyl- $2 H$-tetrazolium bromide (MTT) $\left(2 \mathrm{mg} \mathrm{mL}^{-1}\right)$ was added to each well and incubated at absence of light at $34{ }^{\circ} \mathrm{C}$. After $4 \mathrm{~h}, 150 \mu \mathrm{L}$ of DMSO was added in order to solubilize formazan crystals. The absorbance was read at $570 \mathrm{~nm}$ in microplate reader (Biotek Power Wave XS spectrofluorometer). The concentration that decreased $50 \%\left(\mathrm{CC}_{50}\right)$ of viability of macrophages was determined by linear regression analysis of the data.

\section{Statistical analysis}

The data shown in the tables are expressed as the mean \pm standard deviation of at least three independent experiments. The statistical analysis was performed using GraphPad Prism 6.0 software. ${ }^{40}$ The samples were analyzed using one-way analysis of variance (ANOVA), and the Tukey post hoc test was used to compare means when appropriate. Values of $p \leq 0.05$ were considered statistically significant.

\section{Spin labeling and EPR spectroscopy}

Promastigotes of L. amazonensis in suspension at $5 \times 10^{7}$ parasites $\mathrm{mL}^{-1}(2 \mathrm{~mL})$ were incubated for $2 \mathrm{~h}$ at $26^{\circ} \mathrm{C}$ in culture medium without fetal calf serum (FCS) and containing $150 \mu \mathrm{M}$ of the treatment compound. After incubation, the sample was centrifuged at $1800 \times \mathrm{g}$ for $10 \mathrm{~min}$ to increase the cell concentration to $1 \times 10^{8}$ parasites $\mathrm{mL}^{-1}$ and decrease the final volume to $50 \mu \mathrm{L}$. To incorporate the spin label 5-DSA into the parasite membrane first a spin label film was made on the bottom of a glass tube. An aliquot $(1 \mu \mathrm{L})$ of a 5-DSA ethanolic solution $\left(4 \mathrm{mg} \mathrm{mL}^{-1}\right)$ was added to the tube and after evaporation of ethanol the parasite suspension was placed on the film and stirred gently. Then, the sample was transferred to a 1-mm-i.d. (internal diameter) capillary tube for the EPR measurements.

Spectra were recorded using an EPR EMX-Plus spectrometer of Bruker (Rheinstetten, Germany) with the following spectrometer settings: modulation frequency, $100 \mathrm{kHz}$; modulation amplitude, $1.0 \mathrm{G}$; microwave power, $2 \mathrm{~mW}$; magnetic field scan, $100 \mathrm{G}$; and sample temperature, $25{ }^{\circ} \mathrm{C}$. 


\section{Supplementary Information}

Supplementary information (HRMS, ${ }^{1} \mathrm{H}$ and ${ }^{13} \mathrm{C}$ NMR spectral data) is available free of charge at http://jbcs.sbq.org.br as PDF file.

\section{Acknowledgments}

This work was supported by Fundação Araucária, PR, Brazil (M. H. S., No. 2/2017 Prot. 47223 FA/UEM) and Conselho Nacional de Desenvolvimento Científico e Tecnológico (CNPQ, A. A., 303829/2016-8). We thank CAPES and CNPq for doctoral (P. B.) and postdoctoral (L. A., 150369/2018-2) fellowships, and to Complexo de Centrais de Apoio à Pesquisa of Universidade Estadual de Maringá (COMCAP-UEM) for the facilities.

\section{Author Contributions}

Paula Baréa was responsible for the conceptualization, methodology, data curation, investigation, validation and writing original draft; Jéssica C. de Paula for the methodology, data curation, validation and investigation; Laís Alonso for the methodology, data curation, validation and writing original draft; Aline R. de Oliveira for the methodology and investigation; Willian F. da Costa for the data curation and validation; Antonio Alonso for the formal analysis funding acquisition and resources; Celso V. Nakamura for the investigation, supervision and methodology; Maria $\mathrm{H}$. Sarragiotto for the project administration, conceptualization, formal analysis, funding acquisition, resources, supervision and writing: review and editing.

\section{References}

1. https://www.who.int/leishmaniasis/disease/en/, accessed in December 2019.

2. Torres-Guerrero, E.; Quintanilha-Cedillo, M. R.; RuizEsmenjaud, J.; Arenas, R.; F1000Research 2017, 6, DOI 10.12688/f1000research.11120.1.

3. Kapil, S.; Singh, P. K.; Silakari, O.; Eur. J. Med. Chem. 2018, 157, 339.

4. Modabber, F.; Int. J. Antimicrob. Agents 2010, 36, 58.

5. Chauhan, S. S.; Pandey, S.; Shivahare, R.; Ramalingam, K.; Krishna, S.; Vishwakarma, P.; Siddiqi, M. I.; Gupta, S.; Goyal, N.; Chauhan, P. M. S.; Med. Chem. Commun. 2015, 6, 351.

6. Gohil, V. M.; Brahmbhatt, K. G.; Loiseau, P. M.; Bhutani, K. K.; Bioorg. Med. Chem. Lett. 2012, 22, 3905.

7. Manda, S.; Khan, S. I.; Jain, S. K.; Mohammed, S.; Tekwani, B.; Khan, I. A.; Vishwakarma, R. A.; Bharate, S. B.; Bioorg. Med. Chem. Lett. 2014, 24, 3247.
8. Gellis, A.; Dumètre, A.; Lanzada, G.; Hutter, S.; Ollivier, E.; Vanelle, P.; Azas, N.; Biomed. Pharmacother. 2012, 66, 339.

9. Kumar, A.; Katiyar, S. B.; Gupta, S.; Chauhan, P. M. S.; Eur. J. Med. Chem. 2006, 41, 106.

10. Ashok, P.; Chander, S.; Tejería, A.; García-Calvo, L.; BalanaFouce, R.; Murugesan, S.; Eur. J. Med. Chem. 2016, 123, 814.

11. Ashok, P.; Chander, S.; Chow, L. M. C.; Wong, I. L. K.; Singh, R. P.; Jha, P. N.; Sankaranarayanan, M.; Bioorg. Chem. 2017, 70,100 .

12. Ashok, P.; Chander, S.; Smith, T. K.; Singh, R. P.; Jha, P. N.; Sankaranarayanan, M.; Bioorg. Chem. 2019, 84, 98.

13. Ashok, P.; Chander, S.; Smith, T. K.; Sankaranarayanan, M.; Eur. J. Med. Chem. 2018, 150, 559.

14. Volpato, H.; Desoti, V. C.; Cogo, J.; Panice, M. R.; Sarragiotto, M. H.; Silva, S. O.; Ueda-Nakamura, T.; Nakamura, C. V.; Evid.-Based Complementary Altern. Med. 2013, 2013, DOI $10.1155 / 2013 / 874367$.

15. Silva, C. M. B. L.; Garcia, F. P.; Rodrigues, J. H. S.; Nakamura, C. V.; Ueda-Nakamura, T.; Meyer, E.; Ruiz, A. L. T. G.; Foglio, M. A.; de Carvalho, J. E.; da Costa, W. F.; Sarragiotto, M. H.; Chem. Pharm. Bull. 2012, 60, 1372.

16. Tonin, L. T. D.; Panice, M. R.; Nakamura, C. V.; Rocha, K. J. P.; dos Santos, A. O.; Ueda-Nakamura, T.; da Costa, W. F.; Sarragiotto, M. H.; Biomed. Pharmacother. 2010, 64, 386.

17. Pedroso, R. B.; Tonin, L. T. D.; Ueda-Nakamura, T.; Dias Filho, B. P.; Sarragiotto, M. H.; Nakamura, C. V.; Ann. Trop. Med. Parasitol. 2011, 105, 549.

18. Mendes, E. A.; Desoti, V. C.; Silva, S. O.; Ueda-Nakamura, T.; Dias Filho, B. P.; Yamada-Ogatta, S. F.; Sarragiotto M. H.; Nakamura, C. V.; Chem. Biol. Interact. 2016, 256, 16.

19. Baréa, P.; Barbosa, V. A.; Bidóia, D. L.; de Paula, J. C.; Stefanello, T. F.; da Costa, W. F.; Nakamura, C. V.; Sarragiotto, M. H.; Eur. J. Med. Chem. 2018, 150, 579.

20. Bansal, S.; Halve, A. K.; Int. J. Pharm. Sci. Res. 2014, 5, 4601.

21. Yu, X.; Liu, Y.; Li, Y.; Wang, Q.; J. Agric. Food Chem. 2015, 63, 9690

22. Elarfi, M. J.; Al-Difar, H. A.; Sci. Rev. Chem. Commun. 2012, 2,103 .

23. Sauvaître, T.; Barlier, M.; Herlem, D.; Gresh, N.; Chiaroni, A.; Guenard, D.; Guillou, C.; J. Med. Chem. 2007, 50, 5311.

24. Colotti, G.; Ilari, A.; Fiorillo, A.; Baiocco, P.; Cinellu, M. A.; Maiore, L.; Scaletti, F.; Gabbiani, C.; Messori, L.; ChemMedChem 2013, 8, 1634.

25. Gupta, S.; Yardley, V.; Vishwakarma, P.; Shivahare, R.; Sharma, B.; Launay, D.; Martin, D.; Puri, S. K.; J. Antimicrob. Chemother. 2015, 70, 518.

26. Thompson, A. M.; O'Connor, P. D.; Marshall, A. J.; Yardley, V.; Maes, L.; Gupta, S.; Launay, D.; Braillard, S.; Chatelain, E.; Franzblau, S. G.; Wan, B.; Wang, Y.; Ma, Z.; Cooper, C. B.; Denny, W. A.; J. Med. Chem. 2017, 60, 4212. 
27. Thompson, A. M.; O'Connor, P. D.; Marshall, A. J.; Blaser, A.; Yardley, V.; Maes, L.; Gupta, S.; Launay, D.; Braillard, S.; Chatelain, E.; Wan, B.; Franzblau, S. G.; Ma, Z.; Cooper, C. B.; Denny, W. A.; J. Med. Chem. 2018, 61, 2329.

28. Fernandes, K. S.; de Souza, P. E.; Dorta, M. L.; Alonso, A.; Biochim. Biophys. Acta 2017, 1859, 1.

29. Moreira, R. A.; Mendanha, S. A.; Fernandes, K. S.; Matos, G. G.; Alonso, L.; Dorta, M. L.; Alonso, A.; Antimicrob. Agents Chemother. 2014, 58, 3021.

30. Alonso, L.; Fernandes, K. S.; Mendanha, S. A.; Gonçalves, P. J.; Gomes, R. S.; Dorta, M. L.; Alonso, A.; Biochim. Biophys. Acta 2019, 1861, 1049.

31. Camargos, H. S.; Moreira, R. A.; Mendanha, S. A.; Fernandes, K. S.; Dorta, M. L.; Alonso, A.; PLoS One 2014, 9, e104429.

32. Tiuman, T. S.; Ueda-Nakamura, T.; Alonso, A.; Nakamura, C. V.; BMC Microbiol. 2014, 14, DOI 10.1186/1471-2180-14-152.

33. Desoti, V. C.; Lazarin-Bidóia, D.; Sudatti, D. B.; Pereira, R. C.; Alonso, A.; Ueda-Nakamura, T.; Dias Filho, B. P.; Nakamura, C. V.; Silva, S. O.; Mar. Drugs 2012, 10, 1631.
34. Kaplun, V.; Cogo, J.; Sangi, D. P.; Ueda-Nakamura, T.; Corrêa, A. G.; Nakamura, C. V.; Antimicrob. Agents Chemother. 2016, 60, 02582.

35. Marsh, D.; Eur. Biophys. J. 2010, 39, 513.

36. Mendanha, S. A.; Anjos, J. L. V.; Silva, A. H. M.; Alonso, A.; Braz. J. Med. Biol. Res. 2012, 45, 473.

37. Snyder, L. M.; Fortier, N. L.; Trainor, J.; Jacobs, J.; Leb, L.; Lubin, B.; Chiu, D.; Shohet, S.; Mohandas, N.; J. Clin. Invest. 1985, 76, 1971.

38. Cronin, J. S.; Ginah, F. O.; Murray, A. R.; Copp, J. D.; Synth. Commun. 1996, 26, 3491.

39. Kunishima, M.; Kawachi, C.; Iwasaki, F.; Terao, K.; Tani, S.; Tetrahedron Lett. 1999, 40, 5327.

40. GraphPad Prism 6.0; GraphPad Software Inc., USA, 2013.

Submitted: October 17, 2019 Published online: January 10, 2020 